\title{
Multi-Component One-pot Synthesis of the Tumor-Associated Carbohydrate Antigen Globo-H Based on Pre-activation of Thioglycosyl Donors
}

\author{
Zhen Wang $^{\dagger}$, Luyuan Zhou ${ }^{\dagger}$, Kheireddine El-Boubbou ${ }^{\dagger}$, Xin-shan Ye§, and Xuefei Huang ${ }^{\star}$ \\ $\dagger$ Department of Chemistry, The University of Toledo, 2801 W. Bancroft Street, MS Toledo, Ohio 43606 \\ §The State Key Laboratory of Natural and Biomimetic Drugs, School of Pharmaceutical Sciences, Peking \\ University, Xue Yuan Rd. 38, Beijing 100083, China
}

\begin{abstract}
Two efficient routes for rapid assembly of the tumor-associated carbohydrate antigen Globo- $\mathrm{H}$ hexasaccharide 2 by the pre-activation based iterative one pot strategy are reported. The first method involves the sequential coupling of four glycosyl building blocks, leading to the desired hexasaccharide in $47 \%$ overall yield in one-pot. Although model study on constructing the challenging Gal- $\alpha-1,4-\mathrm{Gal}$ linkage in Gb3 trisaccharide yielded the desired $\alpha$ linkage almost exclusively, similar approach to assemble the hexasaccharide led to formation of significant amount of $\beta$ anomer. As an alternative, the second synthesis utilizes three components in one pot with the Gal- $\alpha-1,4-G a l$ linkage pre-formed, producing the desired hexasaccharide in a similar overall yield as the four component approach. Both methods demonstrate that oligosaccharides containing $\alpha$ and $\beta$ linkages within the same molecule can be constructed in one pot via the pre-activation based approach with higher glyco-assembly efficiencies than the automated solid phase synthesis strategy. Furthermore, because glycosylations can be carried out independent of anomeric reactivities of donors, it is not necessary to differentiate anomeric reactivities of building blocks through extensive protective group adjustment for chemoselective glycosylation. This confers great flexibilities in building block design allowing matching of the donor with the acceptor leading to improved overall yield.
\end{abstract}

\section{Introduction}

Globo-H 1, a member of the globo series glycolipids, is found over-expressed on many types of human cancer cells including breast cancer, prostate cancer, ovarian cancer and lung carcinomas. ${ }^{1-5}$ As a distinct tumor-associated antigenic marker, Globo-H has been conjugated to an immunogenic protein carrier, which is effective in generating protective antibodies against cancer cells. Such a construct has shown promising results in clinical trials as an antibreast cancer vaccine. $^{6-9}$

Due to its biological significance, Globo-H has attracted considerable attention from the synthetic community. It was first assembled by Danishefsky and coworkers using the glycal strategy, 10,11 which was subsequently refined. ${ }^{12}$ Other elegant syntheses include Schmidt's trichloroacetimidate method, ${ }^{13}$ Boon's two-directional glycosylation, ${ }^{14}$ reactivity based onepot method by Wong and coworkers, ${ }^{15-17}$ linear synthesis ${ }^{18}$ and automated solid phase synthesis ${ }^{19}$ by the Seeberger group, as well as syntheses of the non-reducing end fragments. 
20,21 However, many of the reported methods required various synthetic transformations of the oligosaccharide intermediates. Furthermore, with both $\alpha$ and $\beta$ linkages in Globo-H, stereochemical control often became a formidable challenge. ${ }^{10,14,18}$ Therefore, a highly efficient assembly of the Globo-H hexasaccharide is still in great demand.

We have previously developed a pre-activation based iterative one-pot strategy $22-24$ for construction of complex oligosaccharides. One-pot synthesis refers to the glycosylation processes where multiple step glycosylation reactions can be performed in a single reaction flask without synthetic manipulation and purification of intermediate oligosaccharides, thus overcoming a major hurdle in the conventional stepwise chemical synthesis. ${ }^{25}$ For a highyielding one-pot reaction, glycosyl donors and acceptors must be well differentiated, allowing selective donor activation and subsequent glycosylation of the acceptor. This is traditionally accomplished by using building blocks containing different types of activatable aglycons (selective activation), 26,27 or carrying out glycosylations in the order of decreasing anomeric reactivities of glycosyl donors (reactivity-based armed-disarmed approach), ${ }^{28,29}$ or a combination of both strategies. ${ }^{30,31}$ Besides the integration of several glycosylation processes into a single synthetic operation to furnish the target oligosaccharide in a few hours, the advantages of our pre-activation based one-pot approach are 1) only one type of glycosyl donors, i.e., $p$-tolyl thioglycosides are used, thus simplifying the synthetic design; and 2) the pre-activation approach allows us to perform glycosylations without the need to follow decreasing anomeric reactivities of donors, thus granting us much freedom in choosing protective groups to match donors and acceptors. ${ }^{23,32,33}$ As part of the program towards establishing the scope of our method, we explored the synthesis of Globo-H 2 containing both $\alpha$ and $\beta$ linkages by the pre-activation based multi-component one-pot strategy.
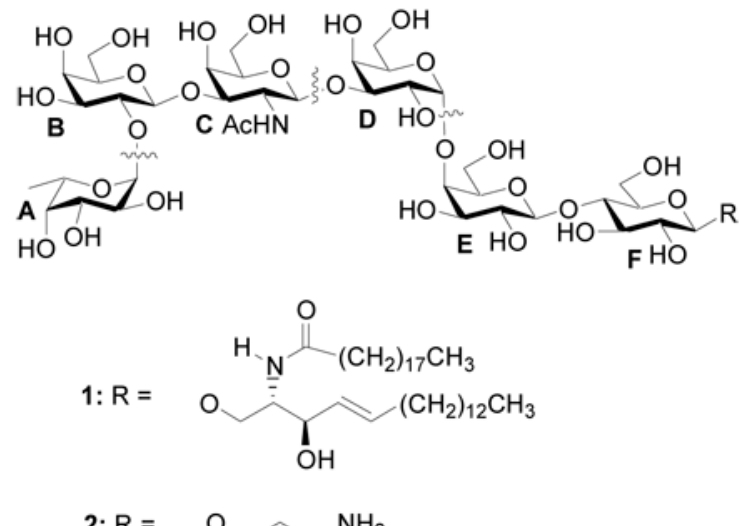

\section{Results and Discussion}

Retrosynthetically, we divided Globo-H into four fragments, A, BC, D and EF. Although the exclusive $\alpha$ linkage between $\mathbf{A}$ and $\mathbf{B}$ units can be produced according to our previous studies, 24 an $\alpha / \beta$ mixture will be formed between the newly formed $\mathbf{A B}$ disaccharide and $\mathbf{C}$ due to the lack of neighboring group participation. ${ }^{14}$ In order to circumvent this difficulty, $\mathbf{B C}$ disaccharide will be prepared in advance with the desired $\beta$ linkage. An aminopropyl spacer will be introduced to the reducing end of Globo-H 2, which can be used for future conjugation to an immunogenic carrier protein. 8,34

The assembly of $\mathbf{B C}$ disaccharide was examined first using several easily prepared glycosyl donor-acceptor pairs and the results are summarized in Table 1. Each glycosylation was carried out by pre-activating the glycosyl donor ${ }^{35}$ in mixed solvents of dichloromethane and diethyl ether using the promoter $p$-TolSOTf, formed in situ by reaction of $p$-TolSCl with AgOTf at 
$-78{ }^{\circ} \mathrm{C},{ }^{36}$ followed by addition of the acceptor. ${ }^{24}$ Reaction of $\mathbf{3}^{37}$ and $4^{37}$ was attempted without success with recovery of acceptor 4 (Table 1, entry 1). Removal of benzylidene in donor $\mathbf{3}$ and replacing the levulinoyl with benzoyl group led to small improvements with recovery of most acceptors (entries 2,3). We investigated next the effect of substituting the bulky phthalimide (Phth) on the acceptor with more sterically accessible trichloroethoxy carbonyl (Troc) and azido moieties. While the Troc group did not have a significant effect (entry 4), glycosylation of azido containing acceptor 6 by donor 8 produced disaccharide $12 \beta\left({ }^{1} \mathrm{H}-\mathrm{NMR}: \delta_{\mathrm{H} 1},=4.79 \mathrm{ppm},{ }^{3} \mathrm{~J}_{\mathrm{H} 1}{ }^{\prime}, \mathrm{H} 2,=7.8 \mathrm{~Hz}\right)$ in $50-70 \%$ yield along with $10-20 \%$ $\mathbf{1 2 \alpha}\left({ }^{1} \mathrm{H}-\mathrm{NMR}: \delta_{\mathrm{H} 1},=5.57 \mathrm{ppm},{ }^{3} \mathrm{~J}_{\mathrm{H} 1^{\prime}, \mathrm{H}{ }^{\prime}}=4.2 \mathrm{~Hz}\right.$ ) (entry 5). The structure of $\mathbf{1 2 \alpha}$ was confirmed by the presence of $\mathrm{Bz}$ carbonyl in the ${ }^{13} \mathrm{C}-\mathrm{NMR}$ spectrum with a chemical shift of $\delta_{\mathrm{Bz}}=166.5 \mathrm{ppm},{ }^{1} \mathrm{~J}_{\mathrm{Cl}}{ }^{\prime}-\mathrm{H} 1^{\prime}=174 \mathrm{~Hz}$ indicating $\alpha$ linakge, ${ }^{38}$ and a HMBC correlation between $\mathrm{C}_{1}$, and $\mathrm{H}_{3}$. The erosion of stereochemical control despite the presence of participating benzoyl group on $\mathrm{C}_{2}$ of the donor is presumably due to the competing $\alpha$-directing effect of ethereal solvents. ${ }^{22,39-44}$ We found that by substituting diethyl ether with small amount of acetonitrile to dissolve AgOTf, the formation of disaccharide $\mathbf{1 2} \alpha$ can be suppressed to negligible amount with $72 \%$ yield of the desired $\beta$ disaccharide $\mathbf{1 2 \beta}$ (entry 6 ). The enhancement of stereoselectivity is most likely due to the exclusion of diethyl ether from the reaction and/or the $\beta$ directing effect of acetonitrile. ${ }^{45}$ The disaccharide $\mathbf{1 2 \beta}$ was then converted to $\mathbf{1 3}$ through standard transformations with an $85 \%$ overall yield (Scheme 1a). The Troc group was introduced to direct the 1,2-trans linkage in future glycosylation.

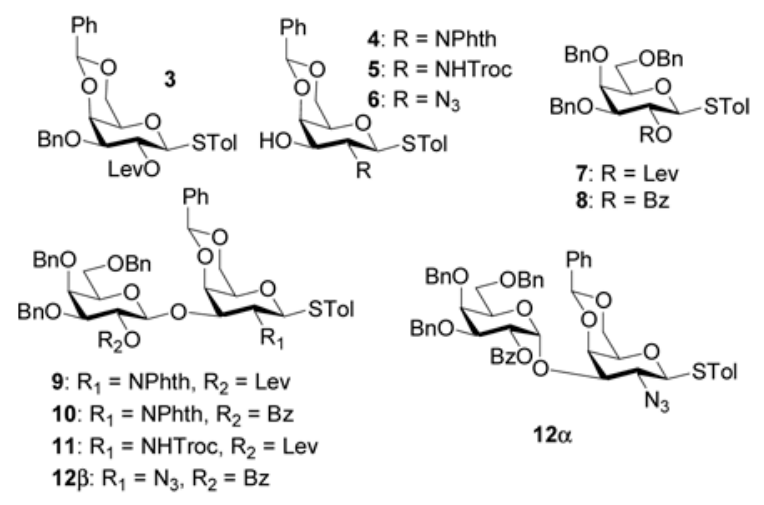

The introduction of the $\alpha-(1-4)$-linkage at the $\mathbf{D E}$ junction of Globo-H is challenging due to the low reactivity of the axial 4-hydroxyl group of the $\mathbf{E}$ ring and the difficulty in stereochemical control. As a model, we explored first the formation of DEF trisaccharide, known as Gb3 or Pk trisaccharide, which is also highly enriched on the surface a variety of cancer cells and involved in many carbohydrate-receptor recognition events. ${ }^{46}$ Many types of glycosyl donors, including fluoride, ${ }^{11,12}$ chloride, ${ }^{46}$ trichloroacetimidate, ${ }^{18,19,47}$ phosphite, ${ }^{48}$ phosphate, 18,19 thioglycoside, ${ }^{49-51}$ sulfoxide ${ }^{52}$ and thioimidates ${ }^{53}$ have been examined in this reaction. The yield for formation of the Gal- $\alpha-(1-4)$-Gal linkage is often not high with anomeric mixture of products. ${ }^{11,18,19,48,50}$ To test whether our glycosylation condition is suitable to construct this key linkage, we examined the glycosylation of lactoside 15 by thiogalactosyl donor 14 (Scheme 1b). Without any optimization, the desired trisaccharide $\mathbf{1 6}$ was obtained in $82 \%$ yield with only trace amount of the $\beta$ anomer isolated. The $\alpha$ stereochemistry of the newly formed glycosidic linkage in $\mathbf{1 6}$ was confirmed by NMR $\left({ }^{1} \mathrm{~J}_{\mathrm{Cl}}\right.$ "- $\mathrm{H} 1$ " $\left.=174 \mathrm{~Hz}\right) .{ }^{38}$ Encouraged by this result, we decided to test the possibility of assembling Globo-H using a four component one pot reactions with building blocks 18, 13, 19 and 15.

Pre-activation of the fucosyl donor $\mathbf{1 8}$ at $-78{ }^{\circ} \mathrm{C}$ with $p$-TolSCl/AgOTf was followed by addition of the first acceptor $\mathbf{1 3}$ (Scheme 2a). A sterically hindered base, 2,4,6-tri-tert-butylpyrimidine (TTBP) ${ }^{54}$ was added with the acceptor to neutralize trifluomethane sulfonic acid 
generated from glycosylation. The reaction temperature was raised to $-20{ }^{\circ} \mathrm{C}$, and the acceptor 13 was completely consumed as judged by TLC analysis. The reaction temperature was cooled back down to $-78^{\circ} \mathrm{C}$, followed by sequential addition of AgOTf, $p$-TolSCl, the second acceptor galactoside 19, TTBP and warming up to $-20^{\circ} \mathrm{C}$. After 19 completely disappeared, the reaction temperature was lowered to $-78^{\circ} \mathrm{C}$ again, and the last acceptor lactoside 15, TTBP, AgOTf, $p$-TolSCl were added to the reaction medium. The fully protected Globo- $\mathrm{H}$ hexasaccharide $20 \boldsymbol{\alpha}$ were obtained in $47 \%$ yield from the four component one-pot reactions within seven hours, which was fully characterized by ${ }^{1} \mathrm{H}-\mathrm{NMR},{ }^{13} \mathrm{C}-\mathrm{NMR}$, gCOSY, gHMQC, gHMBC and HRMS. In addition, the anomer $\mathbf{2 0 \beta}$ was also produced in $23 \%$ yield. ${ }^{55}$ Both Globo- $\mathrm{H}$ anomers will be useful for immunological studies as demonstrated by Danishefsky and coworkers. ${ }^{10}$ It is noteworthy that thiogalactoside $\mathbf{1 9}$ has higher anomeric reactivity than disaccharide $\mathbf{1 3}$. This reversal of anomeric reactivity, i.e., a more reactive thioglycosyl acceptor is glycosylated by a less reactive thioglycosyl donor, is not possible with the reactivity based chemoselective glycosylation method. $28,29,37$ The pre-activation method allowed us to use the readily available building block 19 instead of going through elaborate protective group manipulations to achieve the precise anomeric reactivity. ${ }^{15-17}$

Recently, Seeberger and coworkers have reported an elegant synthesis of Globo-H hexasaccharide ${ }^{19}$ with an overall yield of $30 \%$ for glyco-assembly using the automated solid phase synthesis pioneered by their group. ${ }^{56}$ Compared to the automated method, our synthesis of the desired Globo-H hexasaccharide achieved higher glyco-assembly yield in shorted time without consuming large excess of precious glycosyl building blocks. This underscores the advantage of the pre-activation based iterative one pot oligosaccharide synthesis method.

Deprotection of the hexasaccharide $\mathbf{2 0 \alpha}$ was performed by first removing the Troc protecting group with $1 \mathrm{M} \mathrm{NaOH}$ in THF followed by acetylation. Staudinger reduction of the azide group and subsequent catalytic hydrogenation over Pearlman's catalyst ${ }^{57}$ gave the fully deprotected Globo-H 2 in 50\% overall yield for all deprotection steps (Scheme 2b). Attempts to reduce the azide and remove benzyl groups simultaneously by hydrogenation failed even in the presence of additives such as di- ${ }^{-}$butyl carbonate ${ }^{58}$ and hydrochloric acid.

The formation of $20 \beta$ in the $4+2$ glycosylation is surprising in view of our model studies on $\mathrm{Gb} 3$. Moreover, previous syntheses of Globo-H hexasaccharides through the $4+2$ coupling of tetrasaccharide donors with lactoside acceptors by the Wong group ${ }^{17}$ and the Schmidt group ${ }^{13}$ did not report any stereoisomers being formed. This unpredicted discrepancy highlights the challenge of complex oligosaccharide assembly. The generation of $\mathbf{2 0 \beta}$ may be due to the increased size of the tetrasaccharide donor in our $4+2$ coupling as compared to donor 14. We tested next the lactoside acceptor 21 containing multiple electron withdrawing benzoyl groups in the $4+2$ glycosylation reaction, as the less reactive acceptor is expected to be more selective. However, the glycosylation yield was low with large amount of acceptor recovered.

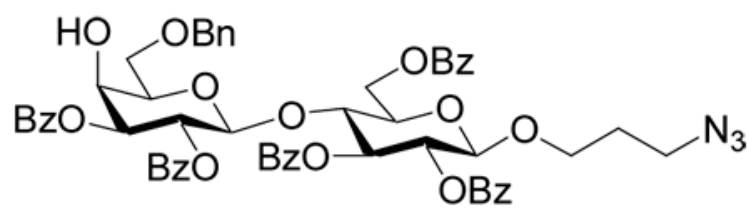

21

Since trisaccharide $\mathbf{1 6}$ was synthesized highly stereoselectively, as an alternative to the four component one-pot strategy, we examined a three component approach with the challenging Gal- $\alpha-1,4-G a l$ linkage preformed. The $p$-methoxybenzyl moiety in trisaccharide $\mathbf{1 6}$ was selectively removed to generate acceptor $\mathbf{1 7}$ in $80 \%$ yield (Scheme 1b). One-pot sequential 
glycosylation of fucoside 18 with disaccharide 13 and trisaccharide 17 produced

hexasaccharide $20 \alpha$ with a $74 \%$ overall yield (Scheme 3), which was identical in all aspects to 20 $\alpha$ prepared via the four component approach thus further confirming our stereochemical assignment. Starting from monosaccharide and disaccharide building blocks, the overall yield for $20 \alpha$ through the three component approach is similar to that of the four component route.

\section{Conclusions}

We have developed two routes for rapid assembly of the tumor-associated carbohydrate antigen Globo-H hexasaccharide 2 by the pre-activation based one pot strategy, demonstrating that oligosaccharides containing $\alpha$ and $\beta$ linkages within the same molecule can be constructed in one pot. Higher glyco-assembly efficiencies have been achieved with only near stoichiometric amount of building blocks via the pre-activation based one-pot method as compared to the automated solid phase synthesis method. However, reliable stereochemical control in glycosylation still remains a challenge, which will require further developments and studies.

\section{Experimental Section}

\section{Characterization of anomeric stereochemistry}

The stereochemistries of the newly formed glycosidic linkages in Globo-H hexasaccharides and intermediates are determined by ${ }^{3} \mathrm{~J}_{\mathrm{H} 1 . \mathrm{H} 2}$ through ${ }^{1} \mathrm{H}-\mathrm{NMR}$ and/or ${ }^{1} \mathrm{~J}_{\mathrm{C} 1, \mathrm{H} 1}$ through gHMQC 2-D NMR (without ${ }^{1} \mathrm{H}$ decoupling). Smaller coupling constants of ${ }^{3} \mathrm{~J}_{\mathrm{H} 1 . \mathrm{H} 2}$ (around $3 \mathrm{~Hz}$ ) indicate $\alpha$ linkages and larger coupling constants ${ }^{3} \mathrm{~J}_{\mathrm{H} 1 . \mathrm{H} 2}(7.2 \mathrm{~Hz}$ or larger) indicate $\beta$ linkages. This can be further confirmed by ${ }^{1} \mathrm{~J}_{\mathrm{C} 1, \mathrm{H} 1}(\sim 170 \mathrm{~Hz})$ for $\alpha$ linkages and ${ }^{1} \mathrm{~J}_{\mathrm{C} 1, \mathrm{H} 1}(\sim 160 \mathrm{~Hz})$ for $\beta$ linkages. 38

\section{p-Tolyl 2-azido-4,6-O-benzylidene-2-deoxy-1-thio- $\beta$-o-galactopyranoside (6)}

Trichloroethoxycarbonyl chloride $(7.7 \mathrm{~mL}, 57.2 \mathrm{mmol})$ was added dropwise over a period of 30 minutes at room temperature to a vigorously stirred solution of $\mathrm{D}_{\mathrm{D}}$ galactosamine hydrochloride (10 g, $46.3 \mathrm{mmol})$ and $\mathrm{NaHCO}_{3}(11.8 \mathrm{~g}, 139.9 \mathrm{mmol})$ in water $(90 \mathrm{~mL})$. The mixture was stirred for another 2 hours and then filtered to give a yellowish solid, which was dried under vacuum. The obtained crude solid $(15 \mathrm{~g})$ was dissolved in pyridine $(50 \mathrm{~mL})$ and then acetic anhydride $(30 \mathrm{~mL})$ was added at $0{ }^{\circ} \mathrm{C}$ over a period of 30 minutes. The mixture was stirred at room temperature under $\mathrm{N}_{2}$ overnight and then quenched with ethanol $(20 \mathrm{~mL})$ at 0 ${ }^{\circ} \mathrm{C}$. The mixture was concentrated and the resulting residue was diluted with ethyl acetate and washed with saturated aqueous solution of $\mathrm{NaHCO}_{3}, 10 \% \mathrm{HCl}$, water, and brine. The organic phase was dried over $\mathrm{Na}_{2} \mathrm{SO}_{4}$, filtered and concentrated. Without separation, the obtained crude solid (21 g) and p-toluenethiol (5.76 g, $46.3 \mathrm{mmol})$ were dissolved in $\mathrm{CH}_{2} \mathrm{Cl}_{2}(60 \mathrm{~mL})$ and the solution was cooled to $0{ }^{\circ} \mathrm{C}$. Boron trifluoride etherate $(17.8 \mathrm{~mL}, 160 \mathrm{mmol})$ was added dropwise at $0^{\circ} \mathrm{C}$ and the mixture was stirred under $\mathrm{N}_{2}$ at room temperature for $6 \mathrm{~h}$. The mixture was diluted with $\mathrm{CH}_{2} \mathrm{Cl}_{2}(400 \mathrm{~mL})$ and washed with saturated aqueous solution of $\mathrm{NaHCO}_{3}$ until the $\mathrm{pH}$ is 7 and then dried over $\mathrm{Na}_{2} \mathrm{SO}_{4}$, filtered and concentrated. The obtained crude product was recrystallized from EtOAc/hexanes to afford compound $p$-tolyl 3,4,6-tri- $O$ acetyl-2-deoxy-2- $N$-trichloroethoxycarbonylamino-1-thio- $\boldsymbol{\beta}$-D-galactopyranoside ${ }^{59}(\mathrm{~S} 1)$ as white solid (20.67 g, 76\% for three steps). ${ }^{1} \mathrm{H}-\mathrm{NMR}\left(600 \mathrm{MHz}, \mathrm{CDCl}_{3}\right): \delta$ 1.98, 2.04, 2.13 (3s, $\left.9 \mathrm{H}, 3 \times \mathrm{COCH}_{3}\right), 2.34$ (s, 3H, SPhCH 3 ), 3.91-3.95 (m, 2H, H-2, H-5), 4.10-4.20 (m, 2H, H-6a, H-6b), 4.73-4.80 (m, 2H, $\left.\mathrm{CH}_{2} \mathrm{CCl}_{3}\right), 4.84\left(\mathrm{~d}, 1 \mathrm{H}, J_{1,2}=10.2 \mathrm{~Hz}, \mathrm{H}-1\right), 5.19\left(\mathrm{dd}, 1 \mathrm{H}, J_{2,3}=\right.$ $\left.10.8 \mathrm{~Hz}, J_{3,4}=2.4 \mathrm{~Hz}, \mathrm{H}-3\right), 5.30\left(\mathrm{~d}, 1 \mathrm{H}, J_{\mathrm{NH}, 2}=9.6 \mathrm{~Hz}, \mathrm{NH}\right), 5.39\left(\mathrm{~d}, 1 \mathrm{H}, J_{3,4}=2.4 \mathrm{~Hz}, \mathrm{H}-4\right)$, 7.10-7.16 (m, 2H), 7.40-7.46 (m, 2H); ${ }^{13} \mathrm{C}-\mathrm{NMR}\left(100 \mathrm{MHz}, \mathrm{CDCl}_{3}\right): \boldsymbol{\delta} 20.9,20.9,21.4,51.4$, 62.0, 67.1, 71.2, 74.6, 74.7, 87.8, 95.7, 128.9, 129.9, 133.3, 138.7, 154.3, 170.5, 170.7, 170.8 . ESI-MS $[\mathrm{M}+\mathrm{Na}]^{+} \mathrm{C}_{22} \mathrm{H}_{26} \mathrm{NaCl}_{3} \mathrm{NO}_{9} \mathrm{~S}$ calcd 608.0, obsd 608.3. $p$-Tolyl 3,4,6-tri-O-acetyl-2deoxy-2- $N$-trichloroethoxycarbonylamino-1-thio- $\beta$-o-galactopyranoside S1 (3 g, $5.1 \mathrm{mmol})$ 
was dissolved in $\mathrm{MeOH}(12 \mathrm{~mL}), \mathrm{AcOH}(6 \mathrm{~mL})$ and $\mathrm{CH}_{2} \mathrm{Cl}_{2}(6 \mathrm{~mL})$. $\mathrm{Zn}$ powder $(6 \mathrm{~g}, 92 \mathrm{mmol})$ was added slowly at $0{ }^{\circ} \mathrm{C}$ and the mixture was stirred under $\mathrm{N}_{2}$ at room temperature for 1 hour. The mixture was filtered and concentrated to dryness. The resulting residue was diluted with $\mathrm{CH}_{2} \mathrm{Cl}_{2}(200 \mathrm{~mL})$ and washed with saturated aqueous solution of $\mathrm{NaHCO}_{3}$ until the $\mathrm{pH}$ is 7 and then dried over $\mathrm{Na}_{2} \mathrm{SO}_{4}$, filtered and concentrated. Silica gel column chromatography (EtOAc) afforded $p$-tolyl 3,4,6-tri- $O$-acetyl-2-amino-2-deoxy-1-thio- $\boldsymbol{\beta}$-р-galactopyranoside as white solid (S2) (1.8 g, 85.7\%). ${ }^{1} \mathrm{H}-\mathrm{NMR}\left(400 \mathrm{MHz}, \mathrm{CDCl}_{3}\right): \boldsymbol{\delta} 2.02,2.05,2.09(3 \mathrm{~s}, 9 \mathrm{H}, 3 \times$ $\left.\mathrm{COCH}_{3}\right), 2.35$ (s, 3H, $\mathrm{SPhCH}_{3}$ ), $3.18(\mathrm{t}, 1 \mathrm{H}, J=10.0 \mathrm{~Hz}, \mathrm{H}-2), 3.91$ (t, $\left.1 \mathrm{H}, J=6.2 \mathrm{~Hz}, \mathrm{H}-5\right)$, 4.11 (dd, $1 \mathrm{H}, J=6.2,10.8 \mathrm{~Hz}, \mathrm{H}-6 \mathrm{a}), 4.18$ (dd, $1 \mathrm{H}, J=6.2,10.8 \mathrm{~Hz}, \mathrm{H}-6 \mathrm{~b}), 4.49$ (d, $1 \mathrm{H}, J_{1,2}$ $=10.0 \mathrm{~Hz}, \mathrm{H}-1), 4.78\left(\mathrm{dd}, 1 \mathrm{H}, J_{2,3}=10.0 \mathrm{~Hz}, J_{3,4}=3.2 \mathrm{~Hz}, \mathrm{H}-3\right), 5.36\left(\mathrm{~d}, 1 \mathrm{H}, J_{3,4}=3.2 \mathrm{~Hz}\right.$, $\mathrm{H}-4), 7.10-7.18$ (m, 2H), 7.44-7.50 (m, 2H); ${ }^{13} \mathrm{C}-\mathrm{NMR}\left(100 \mathrm{MHz}, \mathrm{CDCl}_{3}\right): \delta$ 20.8, 20.9, 20.9, 21.4, 49.9, 62.0, 66.8, 74.4, 75.2, 90.4, 128.4, 129.9, 133.3, 138.6, 170.4, 170.5, 170.7. ESIMS $[\mathrm{M}+\mathrm{H}]^{+} \mathrm{C}_{19} \mathrm{H}_{26} \mathrm{NO}_{7} \mathrm{~S}$ calcd 412.1, obsd 412.1. $p$-Tolyl 3,4,6-tri-O-acetyl-2-amino-2deoxy-1-thio- $\beta$-D-galactopyranoside $\mathrm{S} 2$ (1.8 g, $4.37 \mathrm{mmol}, 1$ equiv) was dissolved in $\mathrm{MeOH}$ (7.5 mL) and $\mathrm{CH}_{2} \mathrm{Cl}_{2}(7.5 \mathrm{~mL}) .1 \mathrm{M} \mathrm{NaOMe}(2.2 \mathrm{~mL}, 2.2 \mathrm{mmol})$ was added and the mixture was stirred at room temperature for $2 \mathrm{~h}$. The mixture was neutralized by conc. $\mathrm{HCl}$ until the $\mathrm{pH}$ is around 7 and then concentrated and dried under vacuum. The resulting residue,

$\mathrm{K}_{2} \mathrm{CO}_{3}(1.5 \mathrm{~g}, 10.87 \mathrm{mmol})$ and catalytic amount of $\mathrm{ZnCl}_{2}(40 \mathrm{mg}, 0.3 \mathrm{mmol})$ were dissolved in $\mathrm{MeOH}(12 \mathrm{~mL})$ and $\mathrm{H}_{2} \mathrm{O}(3 \mathrm{~mL})$. Freshly prepared $\mathrm{TfN}_{3} 60\left(13 \mathrm{~mL}\right.$ in $\left.\mathrm{CH}_{2} \mathrm{Cl}_{2}, 13.1 \mathrm{mmol}\right)$ was added and the mixture was stirred at room temperature overnight. The solvent was evaporated and the resulting residue was diluted with EtOAc $(100 \mathrm{~mL})$. The mixture was neutralized by conc. $\mathrm{HCl}$ until the $\mathrm{pH}$ value is $6-7$ and then concentrated to dryness. Silica gel column chromatography (9:1 $\left.\mathrm{CH}_{2} \mathrm{Cl}_{2}-\mathrm{MeOH}\right)$ afforded $p$-tolyl 2-azido-2-deoxy-1-thio- $\boldsymbol{\beta}$-Dgalactopyranoside ${ }^{61}$ (S3) as a white solid (1.2 g, 88\%). ${ }^{1} \mathrm{H}-\mathrm{NMR}\left(600 \mathrm{MHz}, \mathrm{CD}_{3} \mathrm{OD}\right): \boldsymbol{\delta} 2.22$ (s, 3H, $\mathrm{SPhCH}_{3}$ ), 3.37 (t, $\left.1 \mathrm{H}, J=9.6 \mathrm{~Hz}, \mathrm{H}-2\right), 3.39-3.40$ (m, 2H, H-3, H-5), 3.58-3.68 (m, $2 \mathrm{H}, \mathrm{H}-6 \mathrm{a}, \mathrm{H}-6 \mathrm{~b}), 3.74$ (d, $\left.1 \mathrm{H}, J_{3,4}=2.4 \mathrm{~Hz}, \mathrm{H}-4\right), 4.34\left(\mathrm{~d}, 1 \mathrm{H}, J_{1,2}=9.6 \mathrm{~Hz}, \mathrm{H}-1\right), 7.01-7.06$ (m, 2H), 7.33-7.40 (m, 2H); ${ }^{13} \mathrm{C}-\mathrm{NMR}$ (100 MHz, $\left.\mathrm{CD}_{3} \mathrm{OD}\right): \boldsymbol{\delta} 21.3,62.7,64.6,69.8,75.4$, $80.8,88.5,130.7,130.8,130.9,133.9,134.0,139.3$. ESI-MS $[\mathrm{M}+\mathrm{Na}]^{+} \mathrm{C}_{13} \mathrm{H}_{17} \mathrm{NaN}_{3} \mathrm{O}_{4} \mathrm{~S}$ calcd 334.1 , obsd 334.3. The mixture of compound $p$-tolyl 2-azido-2-deoxy-1-thio- $\beta$-Dgalactopyranoside S3 (1.2 g, $3.85 \mathrm{mmol})$, camphorsulfonic acid $(0.27 \mathrm{~g}, 1.16 \mathrm{mmol})$ and benzaldehyde dimethylacetal $(0.7 \mathrm{~mL}, 4.62 \mathrm{mmol})$ in toluene $(20 \mathrm{~mL})$ was stirred at room temperature for $1 \mathrm{~h}$ and then diluted with EtOAc $(200 \mathrm{~mL})$. The mixture was washed with saturated aqueous solution of $\mathrm{NaHCO}_{3}$, water and then dried over $\mathrm{Na}_{2} \mathrm{SO}_{4}$, filtered and concentrated. Silica gel column chromatography (2:1 Hexanes-EtOAc) afforded compound $\mathbf{6}$ as white solid $(1.15 \mathrm{~g}, 75 \%)$. $[\alpha]_{\mathrm{D}}-31.9\left(c=1, \mathrm{CH}_{2} \mathrm{Cl}_{2}\right) ;{ }^{1} \mathrm{H}-\mathrm{NMR}\left(600 \mathrm{MHz}, \mathrm{CDCl}_{3}\right): \boldsymbol{\delta}$ $2.36\left(\mathrm{~s}, 3 \mathrm{H}, \mathrm{SPhCH}_{3}\right), 2.62\left(\mathrm{~d}, 1 \mathrm{H}, J_{3,3-\mathrm{OH}}=10.2 \mathrm{~Hz}, \mathrm{OH}\right), 3.45(\mathrm{~s}, 1 \mathrm{H}, \mathrm{H}-5), 3.47(\mathrm{t}, 1 \mathrm{H}, J=$ $10.2 \mathrm{~Hz}, \mathrm{H}-2$ ), 3.59 (dt, $\left.1 \mathrm{H}, J_{3,4}=3.0 \mathrm{~Hz} . J_{2,3}=10.2, J_{3,3-\mathrm{OH}}=10.2 \mathrm{~Hz}, \mathrm{H}-3\right), 3.99$ (dd, $1 \mathrm{H}$, $J=1.8,12.6 \mathrm{~Hz}, \mathrm{H}-6 \mathrm{a}), 4.12\left(\mathrm{~d}, 1 \mathrm{H}, J_{3,4}=3.0 \mathrm{~Hz}, \mathrm{H}-4\right), 4.35\left(\mathrm{~d}, 1 \mathrm{H}, J_{1,2}=9.6 \mathrm{~Hz}, \mathrm{H}-1\right), 4.37$ (dd, $1 \mathrm{H}, J=1.8,12.6 \mathrm{~Hz}, \mathrm{H}-6 \mathrm{~b}), 5.49$ (s, $1 \mathrm{H}, \mathrm{CHPh}), 7.11-7.12(\mathrm{~d}, 2 \mathrm{H}, J=7.8 \mathrm{~Hz}$, aromatic), 7.35-7.41 (m, 5H, aromatic), 7.59-7.66 (m, 2H), ${ }^{13} \mathrm{C}-\mathrm{NMR}\left(150 \mathrm{MHz}, \mathrm{CDCl}_{3}\right): \boldsymbol{\delta} 21.6,62.2$, 69.5, 69.96, 70.00, 73.4, 74.6, 74.7, 85.2, 101.6, 101.7, 126.5, 126.8, 128.5, 129.7, 130.1, 135.0, 137.6, 139.0. ESI-MS [M+Na] ${ }^{+} \mathrm{C}_{20} \mathrm{H}_{21} \mathrm{NaO}_{4} \mathrm{~S}$ calcd 422.1, obsd 422.2; Comparison of the NMR data with those reported in the literature ${ }^{37}$ confirmed the identity of 6 .

\section{p-Tolyl 2-O-benzoyl-3,4,6-tri-O-benzyl-1-thio- $\beta$-o-galactopyranoside (8)}

$\boldsymbol{\beta}$-D-Galactopyranosyl pentaacetate $(10 \mathrm{~g}, 25.6 \mathrm{mmol})$ was dissolved in $\mathrm{HBr}$ in acetic acid (30 $\mathrm{mL}, 33 \%$ w/w, $173.6 \mathrm{mmol})$. After 6 hours, the mixture was diluted with $\mathrm{CH}_{2} \mathrm{Cl}_{2}(240 \mathrm{~mL})$ and poured into crushed ice in saturated $\mathrm{NaHCO}_{3}(600 \mathrm{~mL})$. The organic phase was separated and washed again with saturated $\mathrm{NaHCO}_{3}$ until the $\mathrm{pH}$ was about 7 and then dried over $\mathrm{Na}_{2} \mathrm{SO}_{4}$, filtered and concentrated. The resulting residue, 2,6-lutidine (11.93 $\left.\mathrm{mL}, 102.4 \mathrm{mmol}\right)$ and $\mathrm{Bu}_{4} \mathrm{NBr}(3.3 \mathrm{~g}, 10.24 \mathrm{mmol})$ were dissolved in $\mathrm{CH}_{2} \mathrm{Cl}_{2}(45 \mathrm{~mL})$ and dry $\mathrm{EtOH}(8.5 \mathrm{~mL}$, 6 equiv.). The mixture was stirred at room temperature under $\mathrm{N}_{2}$ overnight and then 
concentrated and vacuum dried to afford colorless oil $(10 \mathrm{~g})$. The obtained oil was dissolved in a mixture of $\mathrm{CH}_{2} \mathrm{Cl}_{2} / \mathrm{MeOH}$ (50 mL each) and $1 \mathrm{M}$ solution of $\mathrm{NaOMe}(25.6 \mathrm{~mL}, 25.6 \mathrm{mmol})$ was added at room temperature. The mixture was stirred for $2 \mathrm{~h}$ at room temperature under $\mathrm{N}_{2}$ and then concentrated and vacuum dried. The obtained residue was dissolved in DMF (100 $\mathrm{mL})$ and the solution was cooled to $0{ }^{\circ} \mathrm{C}$. $\mathrm{NaH}(4 \mathrm{~g}, 60 \% \mathrm{NaH}$ in mineral oil, $100 \mathrm{mmol})$ was added in portions, followed by addition of $\mathrm{BnBr}(15 \mathrm{~mL}, 125 \mathrm{mmol})$. The mixture was stirred at room temperature under $\mathrm{N}_{2}$ for 4 hours and then diluted with EtOAc $(300 \mathrm{~mL})$. The mixture was washed with saturated $\mathrm{NaHCO}_{3}$, water and then dried over $\mathrm{Na}_{2} \mathrm{SO}_{4}$, filtered and concentrated. The resulting residue ( $8.5 \mathrm{~g}), p$-toluenethiol $(6.7 \mathrm{~g}, 53.9 \mathrm{mmol})$ and $\mathrm{HgBr}_{2}(0.46$ g, $1.28 \mathrm{mmol})$ were put into $\mathrm{CH}_{3} \mathrm{CN}(20 \mathrm{~mL})$ and the mixture was heated at $60{ }^{\circ} \mathrm{C}$ under $\mathrm{N}_{2}$ overnight. The solvent was evaporated and then the residue was diluted with $\mathrm{CH}_{2} \mathrm{Cl}_{2}(300 \mathrm{~mL})$. The mixture was washed with saturated $\mathrm{NaHCO}_{3}$, water and $10 \% \mathrm{HCl}$ and then dried over $\mathrm{Na}_{2} \mathrm{SO}_{4}$, filtered and concentrated. Silica gel column chromatography (2:1 Hexanes-EtOAc) afforded compound $p$-tolyl 2-O-acetyl-3,4,6-tri- $O$-benzyl-1-thio- $\beta$-d-galactopyranoside ${ }^{62}$ (S4) as white solid (6.4 g, $42 \%$ for 5 steps). ${ }^{1} \mathrm{H}-\mathrm{NMR}\left(600 \mathrm{MHz}, \mathrm{CDCl}_{3}\right): \boldsymbol{\delta} 2.04$ (s, 3H, $\left.\mathrm{COCH}_{3}\right), 2.29\left(\mathrm{~s}, 3 \mathrm{H}, \mathrm{SPhCH}_{3}\right), 3.54\left(\mathrm{dd}, 1 \mathrm{H}, J_{2,3}=9.6 \mathrm{~Hz}, J_{3,4}=3.0 \mathrm{~Hz}, \mathrm{H}-3\right), 3.59-3.67$ (m, 3H, H-5, H-6a, H-6b), 3.97 (d, 1H, $\left.J_{3,4}=3.0 \mathrm{~Hz}, \mathrm{H}-4\right), 4.41,4.45,4.52,4.56$ (4d, 4H, $J=$ $\left.12.0 \mathrm{~Hz}, \mathrm{CH}_{2} \mathrm{Ph}\right), 4.55$ (d, $\left.1 \mathrm{H}, J_{1,2}=9.6 \mathrm{~Hz}, \mathrm{H}-1\right), 4.66\left(\mathrm{~d}, 1 \mathrm{H}, J=12.0 \mathrm{~Hz}, \mathrm{CH}_{2} \mathrm{Ph}\right), 4.93(\mathrm{~d}$, $\left.1 \mathrm{H}, J=12.0 \mathrm{~Hz}, \mathrm{CH}_{2} \mathrm{Ph}\right), 5.39(\mathrm{t}, 1 \mathrm{H}, J=9.6 \mathrm{~Hz}, \mathrm{H}-2), 6.98-7.06(\mathrm{~m}, 2 \mathrm{H}), 7.25-7.34(\mathrm{~m}, 15 \mathrm{H}$, aromatic), 7.37-7.38 (m, 2H); ${ }^{13} \mathrm{C}-\mathrm{NMR}\left(150 \mathrm{MHz}, \mathrm{CDCl}_{3}\right): \boldsymbol{\delta} 21.3,21.4,69.0,70.0,72.2$, 73.0, 73.8, 74.6, 77.8, 81.7, 87.3, 127.67, 127.70, 127.99, 128.03, 128.1, 128.2, 128.4, 128.6, 129.7, 130.0, 132.7, 137.8, 138.09, 138.12, 138.7, 169.7. ESI-MS $[\mathrm{M}+\mathrm{Na}]^{+} \mathrm{C}_{36} \mathrm{H}_{38} \mathrm{NaO}_{6} \mathrm{~S}$ calcd 621.2, obsd 621.5. $p$-Tolyl 2- $O$-acetyl-3,4,6-tri- $O$-benzyl-1-thio- $\beta$-o-galactopyranoside $\mathrm{S} 4(3 \mathrm{~g}, 5 \mathrm{mmol})$ was dissolved in a mixture of $\mathrm{CH}_{2} \mathrm{Cl}_{2} / \mathrm{MeOH}(50 \mathrm{~mL}$ each) and $1 \mathrm{M} \mathrm{NaOMe}$ $(10 \mathrm{~mL}, 10 \mathrm{mmol})$ was added at room temperature. The mixture was stirred for $2 \mathrm{~h}$ at room temperature under $\mathrm{N}_{2}$ and then was neutralized with Amberlite IR-120 until the $\mathrm{pH}$ is around 6-7. The mixture was concentrated and diluted with $\mathrm{CH}_{2} \mathrm{Cl}_{2}(300 \mathrm{~mL})$ and washed with saturated $\mathrm{NaHCO}_{3}, 10 \% \mathrm{HCl}$ and water and then dried over $\mathrm{Na}_{2} \mathrm{SO}_{4}$, filtered and concentrated. Silica gel column chromatography (2:1 Hexanes-EtOAc) afforded compound $p$-tolyl 3,4,6-tri$O$-benzyl-1-thio- $\boldsymbol{\beta}$-D-galactopyranoside (S5) as white solid (2.56 g, 92\%). ${ }^{1} \mathrm{HNMR}(600 \mathrm{MHz}$, $\left.\mathrm{CDCl}_{3}\right): \delta 2.29\left(\mathrm{~s}, 3 \mathrm{H}, \mathrm{SPhCH}_{3}\right), 2.42\left(\mathrm{~d}, 1 \mathrm{H}, J_{2,2-\mathrm{OH}}=2.4 \mathrm{~Hz}, \mathrm{OH}\right), 3.46\left(\mathrm{dd}, 1 \mathrm{H}, J_{2,3}=9.6\right.$ $\left.\mathrm{Hz}, J_{3,4}=2.4 \mathrm{~Hz}, \mathrm{H}-3\right), 3.63-3.66$ (m, 3H, H-5, H-6a, H-6b), 3.95-3.98 (m, 2H, H-2, H-4), $4.44\left(\mathrm{~d}, 1 \mathrm{H}, J=12.0 \mathrm{~Hz}, \mathrm{CH}_{2} \mathrm{Ph}\right), 4.47$ (d, $\left.1 \mathrm{H}, J_{1,2}=9.6 \mathrm{~Hz}, \mathrm{H}-1\right), 4.49,4.56,4.67,4.73,4.89$ (5d, $\left.5 \mathrm{H}, J=12.0 \mathrm{~Hz}, \mathrm{CH}_{2} \mathrm{Ph}\right), 6.98-7.05(\mathrm{~m}, 2 \mathrm{H}), 7.25-7.35$ (m, 15H, aromatic), 7.44-7.45 (m, 2H); ${ }^{13} \mathrm{C}-\mathrm{NMR}\left(150 \mathrm{MHz}, \mathrm{CDCl}_{3}\right): \delta$ 21.4, 68.9, 69.3, 72.6, 73.5, 73.8, 74.6, 77.8, 83.4, 89.0, 127.6, 127.9, 127.95, 128.04, 128.05, 128.2, 128.4, 128.7, 128.8, 128.9, 129.8, 133.1, 138.0, 138.1, 138.3, 138.9. ESI-MS $[\mathrm{M}+\mathrm{Na}]^{+} \mathrm{C}_{34} \mathrm{H}_{36} \mathrm{NaO}_{5} \mathrm{~S}$ calcd 579.2, obsd 579.6. $p$-Tolyl 3,4,6-tri- $O$-benzyl-1-thio- $\beta$-D-galactopyranoside $55(3.8 \mathrm{~g}, 6.8 \mathrm{mmol})$ and $N, N$-dimethylamino pyridine (DMAP) $(0.08 \mathrm{~g}, 0.68 \mathrm{mmol})$ were dissolved in pyridine $(20 \mathrm{~mL})$ and then benzoyl chloride ( $2.38 \mathrm{~mL}, 20.5 \mathrm{mmol}$ ) was added. The mixture was stirred at room temperature under $\mathrm{N}_{2}$ for $4 \mathrm{~h}$ and then diluted with of $\mathrm{CH}_{2} \mathrm{Cl}_{2}(200 \mathrm{~mL})$. The mixture was washed with saturated $\mathrm{NaHCO}_{3}$, water and $10 \% \mathrm{HCl}$ and then dried over $\mathrm{Na}_{2} \mathrm{SO}_{4}$, filtered and concentrated. Silica gel column chromatography (2:1 Hexanes-EtOAc) afforded compound $\mathbf{8}$ as white solid (4.2 g, 93\%). $[\alpha]_{\mathrm{D}}+38.2\left(c=1, \mathrm{CH}_{2} \mathrm{Cl}_{2}\right) ;{ }^{1} \mathrm{H}-\mathrm{NMR}\left(600 \mathrm{MHz}, \mathrm{CDCl}_{3}\right): \boldsymbol{\delta} 2.25\left(\mathrm{~s}, 3 \mathrm{H}, \mathrm{SPhCH}_{3}\right)$, 3.65-3.69 (m, 4H, H-3, H-5, H-6a, H-6b), 4.03 (d, 1H, J $J_{3,4}=2.4$ Hz, H-4), 4.41, 4.45, 4.47, 4.59, $4.61\left(5 \mathrm{~d}, 5 \mathrm{H}, J=12.0 \mathrm{~Hz}, \mathrm{CH}_{2} \mathrm{Ph}\right), 4.71\left(\mathrm{~d}, 1 \mathrm{H}, J_{1,2}=10.2 \mathrm{~Hz}, \mathrm{H}-1\right), 4.97(\mathrm{~d}, 1 \mathrm{H}, J=$ $\left.12.0 \mathrm{~Hz}, \mathrm{CH}_{2} \mathrm{Ph}\right), 5.68(\mathrm{t}, 1 \mathrm{H}, J=10.2 \mathrm{~Hz}, \mathrm{H}-2), 6.96-6.97$ (d, $2 \mathrm{H}, J=7.8 \mathrm{~Hz}$, aromatic), 7.107.15 (m, 5H, aromatic), 7.25-7.36 (m, 12H, aromatic), 7.41-7.42 (m, 2H, aromatic), 7.53-7.55 (m, 1H, aromatic), 8.00-8.07 (m, 2H); ${ }^{13} \mathrm{C}-\mathrm{NMR}\left(150 \mathrm{MHz}, \mathrm{CDCl}_{3}\right): \delta$ 21.5, 69.2, 70.8, 72.1, 73.1, 73.9, 74.7, 78.0, 81.5, 87.5, 127.8, 127.99, 128.00, 128.1, 128.2, 128.4, 128.5, 128.6, $128.7,128.8,129.8,130.0,130.2,130.5,133.0,133.4,137.9,138.0,138.2,138.8,165.6$. HRMS: $[\mathrm{M}+\mathrm{Na}]^{+} \mathrm{C}_{41} \mathrm{H}_{40} \mathrm{NaO}_{6} \mathrm{~S}$ calcd 683.2443, obsd 683.2421. 


\section{General procedure for single step pre-activation based glycosylation}

A solution of donor $(0.060 \mathrm{mmol})$ and freshly activated molecular sieve MS $4 \AA$ ( $200 \mathrm{mg})$ in $\mathrm{CH}_{2} \mathrm{Cl}_{2}(2 \mathrm{~mL})$ was stirred at room temperature for 30 minutes, and cooled to $-78{ }^{\circ} \mathrm{C}$, which was followed by addition of AgOTf (47 mg, $0.18 \mathrm{mmol}$ ) dissolved in $\mathrm{Et}_{2} \mathrm{O}(1 \mathrm{~mL})$ without touching the wall of the flask. After 5 minutes, orange colored $p$-TolSCl $(9.5 \mu \mathrm{L}, 0.060 \mathrm{mmol})$ was added through a microsyringe. Since the reaction temperature was lower than the freezing point of $p$-TolSCl, $p$-TolSCl was added directly into the reaction mixture to prevent it from freezing on the flask wall. The characteristic yellow color of $p$-TolSCl in the reaction solution dissipated rapidly within a few seconds indicating depletion of $p$-TolSCl. After the donor was completely consumed according to TLC analysis (about 5 minutes at $-78{ }^{\circ} \mathrm{C}$ ), a solution of acceptor $(0.060 \mathrm{mmol})$ in $\mathrm{CH}_{2} \mathrm{Cl}_{2}(0.2 \mathrm{~mL})$ was slowly added dropwise via a syringe. The reaction mixture was warmed to $-10{ }^{\circ} \mathrm{C}$ under stirring in 2 hours. Then the mixture was diluted with $\mathrm{CH}_{2} \mathrm{Cl}_{2}(20 \mathrm{~mL})$ and filtered over Celite. The Celite was further washed with $\mathrm{CH}_{2} \mathrm{Cl}_{2}$ until no organic compounds were observed in the filtrate by TLC. All $\mathrm{CH}_{2} \mathrm{Cl}_{2}$ solutions were combined and washed twice with saturated aqueous solution of $\mathrm{NaHCO}_{3}(20 \mathrm{~mL})$ and twice with water $(10 \mathrm{~mL})$. The organic layer was collected and dried over $\mathrm{Na}_{2} \mathrm{SO}_{4}$. After removal of the solvent, the desired disaccharide was purified from the reaction mixture via silica gel flash chromatography.

\section{$p$-Tolyl 2-O-benzoyl-3,4,6-tri-O-benzyl- $\beta$-o-galactopyranosyl-(1 $\rightarrow 3)-2-a z i d o-4,6-O$ - benzylidene-2-deoxy-1-thio- $\beta$-o-galactopyranoside (12ß) and $p$-tolyl 2-O-benzoyl-3,4,6-tri- $O$ - benzyl- $\alpha$-o-galactopyranosyl-(1 $\rightarrow 3)$-2-azido-4,6-O-benzylidene-2-deoxy-1-thio- $\beta$-o- galactopyranoside $(12 \alpha)$}

Compound $\mathbf{1 2 \beta}$ and $\mathbf{1 2} \alpha$ was synthesized from donor $\mathbf{8}$ and acceptor $\mathbf{6}$ in 50-70\% and 10-20\% yield respectively following the general procedure of single step glycosylation. When $\mathrm{CH}_{3} \mathrm{CN}$ $(100 \mu \mathrm{l})$ was used instead of diethyl ether to dissolve the AgOTf, the formation of disaccharide $12 \alpha$ can be suppressed to negligible amount with $72 \%$ yield of the desired $\beta$ disaccharide

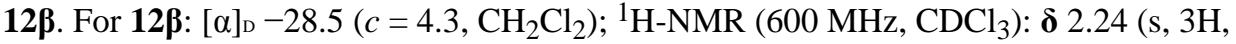
$\mathrm{SPhCH}_{3}$ ), 3.31 (s, 1H, H-5), 3.55-3.65 (m, 6H, H-2, H-3, H-3',H-5',H-6a', H-6b'), 3.84 (d, $1 \mathrm{H}, J=10.8 \mathrm{~Hz}, \mathrm{H}-6 \mathrm{a}$ ), 3.96 (d, 1H, $J_{3^{\prime}, 4^{\prime}}=2.4 \mathrm{~Hz}, \mathrm{H}-4^{\prime}$ ), 4.24-4.27 (m, 2H, H-4, H-6b), 4.30 $\left(\mathrm{d}, 1 \mathrm{H}, J_{1,2}=9.6 \mathrm{~Hz}, \mathrm{H}-1\right), 4.44-4.47\left(\mathrm{~m}, 3 \mathrm{H}, \mathrm{CH}_{2} \mathrm{Ph}\right), 4.59-4.62(\mathrm{~m}, 2 \mathrm{H}, J=12.0 \mathrm{~Hz}$, $\left.\mathrm{CH}_{2} \mathrm{Ph}\right), 4.79\left(\mathrm{~d}, 1 \mathrm{H}, J_{1^{\prime}, 2^{\prime}}=7.8 \mathrm{~Hz}, \mathrm{H}-1^{\prime}\right), 4.98\left(\mathrm{~d}, 1 \mathrm{H}, J=12.0 \mathrm{~Hz}, \mathrm{CH}_{2} \mathrm{Ph}\right), 5.37(\mathrm{~s}, 1 \mathrm{H}$, CHPh), 5.62 (t, 1H, $\left.J_{1^{\prime}, 2^{\prime}}=7.8 \mathrm{~Hz}, \mathrm{H}-2^{\prime}\right), 6.83-6.92(\mathrm{~m}, 2 \mathrm{H}), 7.10-7.18$ (m, 5H, aromatic), 7.23-7.41 (m, 17H, aromatic), 7.43-7.52 (m, 2H), 7.49-7.59 (m, 1H), 7.94-8.04 (m, $2 \mathrm{H}) ;{ }^{13} \mathrm{C}-\mathrm{NMR}\left(150 \mathrm{MHz}, \mathrm{CDCl}_{3}\right): \boldsymbol{\delta} 21.4,59.7,69.2,69.3,70.1,71.7,72.0,72.6,73.7,74.2$, $74.6,74.9,79.3,80.3,85.8,100.8,102.3,126.2,126.7,127.8,127.9,128.0,128.1,128.2,128.4$, 128.5, 128.6, 128.7, 128.9, 129.9, 130.0, 130.2, 133.1, 134.5, 137.6, 137.9, 138.0, 138.5, 138.6, 165.5; HRMS: $[\mathrm{M}+\mathrm{Na}]^{+} \mathrm{C}_{54} \mathrm{H}_{53} \mathrm{~N}_{3} \mathrm{NaO}_{10} \mathrm{~S}$ calcd 958.3349, obsd 958.3365. gHMQC (without ${ }^{1} \mathrm{H}$ decoupling): ${ }^{1} \mathrm{~J}_{\mathrm{Cl}}{ }^{\prime}, \mathrm{H} 1{ }^{\prime}=160.9 \mathrm{~Hz},{ }^{1} \mathrm{~J}_{\mathrm{C} 1, \mathrm{H} 1}=159.9 \mathrm{~Hz}$; For $12 \alpha$ : $[\alpha]_{\mathrm{D}}+70.8(c=$ 4.5, $\mathrm{CH}_{2} \mathrm{Cl}_{2}$ ); ${ }^{1} \mathrm{H}-\mathrm{NMR}\left(600 \mathrm{MHz}, \mathrm{CDCl}_{3}\right): \boldsymbol{\delta} 2.27$ (s, 3H, $\mathrm{SPhCH}_{3}$ ), 3.26 (s, $\left.1 \mathrm{H}, \mathrm{H}-5\right), 3.57-$ 3.65 (m, 3H, H-3, H-6a', H-6b'), 3.79 (t, 1H, J = 9.6 Hz, H-2), 3.86 (d, 1H, J = $12.0 \mathrm{~Hz}, \mathrm{H}-6 \mathrm{a}$ ), $4.06(\mathrm{~s}, 1 \mathrm{H}, \mathrm{H}-4), 4.09\left(\mathrm{~d}, 1 \mathrm{H}, J_{3^{\prime}, 4^{\prime}}=2.4 \mathrm{~Hz}, \mathrm{H}^{\prime} 4^{\prime}\right), 4.17\left(\mathrm{dd}, 1 \mathrm{H}, J_{2^{\prime}, 3^{\prime}}=10.8 \mathrm{~Hz}, J_{3^{\prime}, 4^{\prime}}=2.4\right.$ Hz, H-3'), 4.22-4.24 (m, 2H, H-5', H-6b), 4.32 (d, 1H, J1,2 = 9.6 Hz, H-1), 4.47, 4.51, 4.59, 4.66, 4.68, $4.93\left(6 \mathrm{~d}, 6 \mathrm{H}, J=12.0 \mathrm{~Hz}, \mathrm{CH}_{2} \mathrm{Ph}\right), 5.16(\mathrm{~s}, 1 \mathrm{H}, \mathrm{CHPh}), 5.42\left(\mathrm{dd}, 1 \mathrm{H}, J_{1}{ }^{\prime}, 2^{\prime}=3.6\right.$ $\left.\mathrm{Hz}, J_{2}{ }^{\prime} 3^{\prime}=10.8 \mathrm{~Hz}, \mathrm{H}-2^{\prime}\right), 5.57\left(\mathrm{~d}, 1 \mathrm{H}, J_{1^{\prime}, 2^{\prime}}=3.6 \mathrm{~Hz}, \mathrm{H}-1^{\prime}\right), 6.90-6.99(\mathrm{~m}, 2 \mathrm{H}), 6.98-7.02$ (m, 4H, aromatic), 7.12-7.44 (m, 19H, aromatic), 7.50-7.59 (m, 2H), 7.72-7.82 (m, $2 \mathrm{H}) ;{ }^{13} \mathrm{C}-\mathrm{NMR}\left(150 \mathrm{MHz}, \mathrm{CDCl}_{3}\right): \boldsymbol{\delta} 21.4,59.6,68.9,69.2,69.7,70.0,70.7,71.9,73.2,73.4$, 74.7, 75.0, 76.0, 76.9, 85.7, 93.1, 100.2, 126.1, 126.9, 127.78, 127.80, 127.82, 127.86, 127.87, $128.3,128.45,128.49,128.51,128.55,129.68,129.70,129.9,133.0,134.3,137.5,138.2$, 138.3, 138.4, 138.5, 166.5. ESI-MS $[\mathrm{M}+\mathrm{Na}]^{+} \mathrm{C}_{54} \mathrm{H}_{53} \mathrm{~N}_{3} \mathrm{NaO}_{10} \mathrm{~S}$ calcd 958.3, obsd 958.4. gHMQC (without ${ }^{1} \mathrm{H}$ decoupling): ${ }^{1} \mathrm{~J}_{\mathrm{Cl}}{ }^{\prime}, \mathrm{H} 1{ }^{\prime}=172.4 \mathrm{~Hz},{ }^{1} \mathrm{~J}_{\mathrm{C} 1, \mathrm{H} 1}=160.1 \mathrm{~Hz}$. 


\section{$p$-Tolyl 3,4,6-tri-O-benzyl- $\beta$-o-galactopyranosyl-(1 $\rightarrow 3)-4,6$-benzylidene-2-(2,2,2- trichloroethoxycarbonylamino)-2-deoxy-1-thio- $\beta$-o-galactopyranoside (13)}

Compound $\mathbf{1 2 \beta}$ (1.7 g, $1.81 \mathrm{mmol}$ ) was dissolved in a mixture of $\mathrm{CH}_{2} \mathrm{Cl}_{2} / \mathrm{MeOH}(25 \mathrm{~mL}$ each) and $1 \mathrm{M} \mathrm{NaOMe}(5.4 \mathrm{~mL}, 5.4 \mathrm{mmol})$ was added at room temperature. The mixture was heated at reflux for $4 \mathrm{~h}$ under $\mathrm{N}_{2}$ and then was neutralized with conc. $\mathrm{HCl}$ until the $\mathrm{pH}$ is around 7. The mixture was concentrated and then diluted with of $\mathrm{CH}_{2} \mathrm{Cl}_{2}(200 \mathrm{~mL})$. The organic phase was washed with saturated aqueous solution of $\mathrm{NaHCO}_{3}, 10 \% \mathrm{HCl}$ and water and then dried over $\mathrm{Na}_{2} \mathrm{SO}_{4}$, filtered and concentrated. Silica gel column chromatography $(3: 1: 1$ HexanesEtOAc- $\mathrm{CH}_{2} \mathrm{Cl}_{2}$ ) afforded $p$-tolyl 3,4,6-tri- $O$-benzyl- $\boldsymbol{\beta}$-d-galactopyranosyl- $(1 \rightarrow 3)-4,6$ benzylidene-2-azido-2-deoxy-1-thio- $\beta$-D-galactopyranoside (S6) as white solid (1.5 g, quantitative). ${ }^{1} \mathrm{H}-\mathrm{NMR}\left(600 \mathrm{MHz}, \mathrm{CDCl}_{3}\right): \boldsymbol{\delta} 2.32\left(\mathrm{~s}, 3 \mathrm{H}, \mathrm{SPhCH}_{3}\right), 2.52$ (s, 1H, OH), 3.35 (s, br, 1H, H-5), 3.46 (dd, $\left.1 \mathrm{H}, J_{2}{ }^{\prime}, 3^{\prime}=9.6 \mathrm{~Hz}, J_{3}{ }^{\prime}, 4^{\prime}=3.0 \mathrm{~Hz}, \mathrm{H}-3{ }^{\prime}\right), 3.51-3.61$ (m, 4H, H-3, H-5', H-6a', H-6b'), 3.78 (dd, 1H, $J_{1,2}=10.2$ Hz, H-2), 3.85 (d, 1H, J $3.4=3.0$ Hz, H-4'), 3.863.89 (dd, $1 \mathrm{H}, J=1.8,12.0 \mathrm{~Hz}, \mathrm{H}-6 \mathrm{a}), 3.96$ (t, $1 \mathrm{H}, J=9.6 \mathrm{~Hz}, \mathrm{H}-2$ '), 4.22 (d, 1H, J ', ', = 3.0 $\mathrm{Hz}, \mathrm{H}-4), 4.31$ (dd, $1 \mathrm{H}, J=1.8,12.0 \mathrm{~Hz}, \mathrm{H}-6 \mathrm{~b}), 4.35$ (d, $1 \mathrm{H}, J_{1,2}=10.2 \mathrm{~Hz}, \mathrm{H}-1$ ), $4.42,4.46$ $\left(2 \mathrm{~d}, 2 \mathrm{H}, J=12.0 \mathrm{~Hz}, \mathrm{CH}_{2} \mathrm{Ph}\right), 4.48$ (d, $1 \mathrm{H}, J_{1}{ }^{\prime}, 2$ ' = 9.6 Hz, H-1'), 4.59, 4.72, 4.77, 4.90 (4d, $\left.4 \mathrm{H}, \mathrm{CH}_{2} \mathrm{Ph}\right), 5.45$ (s, 1H, CHPh), 6.98-7.08 (m, 2H), 7.25-7.43 (m, 20H, aromatic), 7.58-7.68 $(\mathrm{m}, 2 \mathrm{H}) ;{ }^{13} \mathrm{C}-\mathrm{NMR}\left(150 \mathrm{MHz}, \mathrm{CDCl}_{3}\right): \boldsymbol{\delta} 21.5,60.2,69.3,69.4,70.3,71.4,73.3,73.6,73.7$, 74.3, 74.9, 75.4, 80.4, 82.1, 85.7, 101.3, 105.4, 126.2, 127.0, 127.9, 128.98, 127.99, 128.2, $128.3,128.5,128.6,128.7,128.8,129.3,130.1,135.0,138.0,138.1,138.5,138.6,138.9$; HRMS: $[\mathrm{M}+\mathrm{Na}]^{+} \mathrm{C}_{47} \mathrm{H}_{49} \mathrm{~N}_{3} \mathrm{NaO}_{9} \mathrm{~S}$ calcd 854.3087, obsd 854.3085. $p$-Tolyl 3,4,6-tri- $O$ benzyl- $\boldsymbol{\beta}$-D-galactopyranosyl-( $1 \rightarrow 3)$-4,6-benzylidene-2-azido-2-deoxy-1-thio- $\boldsymbol{\beta}$-рgalactopyranoside $\mathrm{S} 6$ (1.3 g, $1.56 \mathrm{mmol}), 1,3$-propanedithiol $(1.57 \mathrm{~mL}, 15.6 \mathrm{mmol})$ and $\mathrm{Et}_{3} \mathrm{~N}$ $(1.10 \mathrm{~mL}, 15.6 \mathrm{mmol})$ were dissolved in a mixture of $\mathrm{CH}_{2} \mathrm{Cl}_{2} / \mathrm{MeOH}$ (10 mL each). The mixture was heated at reflux overnight under $\mathrm{N}_{2}$ and then concentrated. The resulting residue was diluted with $\mathrm{CH}_{2} \mathrm{Cl}_{2}(200 \mathrm{~mL})$ and then washed with saturated aqueous solution of $\mathrm{NaHCO}_{3}$ and water, dried over $\mathrm{Na}_{2} \mathrm{SO}_{4}$, filtered and concentrated. Silica gel column chromatography (20:1 $\left.\mathrm{CH}_{2} \mathrm{Cl}_{2}-\mathrm{MeOH}\right)$ afforded $p$-tolyl 3,4,6-tri- $O$-benzyl- $\boldsymbol{\beta}$-Dgalactopyranosyl-( $\rightarrow 3$ )-4,6-benzylidene-2-amino-2-deoxy-1-thio- $\boldsymbol{\beta}$-D-galactopyranoside (S7) as white solid (1.07 g, 85\%). ${ }^{1} \mathrm{H}-\mathrm{NMR}\left(600 \mathrm{MHz}, \mathrm{CDCl}_{3}\right): \boldsymbol{\delta} 2.32\left(\mathrm{~s}, 4 \mathrm{H}, \mathrm{SPhCH}_{3}, \mathrm{OH}\right)$, 3.25 (t, $1 \mathrm{H}, J=9.6 \mathrm{~Hz}, \mathrm{H}-2), 3.37$ (s, $1 \mathrm{H}, \mathrm{H}-5), 3.37-3.39$ (dd, $1 \mathrm{H}, J_{2}{ }^{\prime}, 3^{\prime}=9.6 \mathrm{~Hz}, J_{3}{ }^{\prime}, 4^{\prime}=3.0$ Hz, H-3'), 3.53 (dd, 1H, $J_{2,3}=10.2$ Hz, $J_{3,4}=3.0$ Hz, H-3), 3.55-3.59 (m, 3H, H-5', H-6a', H-6b'), 3.86 (d, 1H, J' ', ' $=3.0 \mathrm{~Hz}, \mathrm{H}-4^{\prime}$ ), 3.86 (d, 1H, J=12.0 Hz, H-6a), 3.95 (t, 1H, J = 9.6 Hz, H-2'), 4.16 (d, 1H, J $\left.J_{3.4}=3.0 \mathrm{~Hz}, \mathrm{H}-4\right), 4.29$ (d, 1H, J = $\left.12.0 \mathrm{~Hz}, \mathrm{H}-6 \mathrm{~b}\right), 4.35$ (d, 1H, $J_{1,2}$ $=9.6 \mathrm{~Hz}, \mathrm{H}-1)$, 4.43-4.44 (m, 3H, $\left.J_{1}{ }^{\prime},{ }^{\prime}{ }^{\prime}=10.2 \mathrm{~Hz}, \mathrm{H}-1^{\prime}, \mathrm{CH}_{2} \mathrm{Ph}\right), 4.57,4.65,4.68,4.86$ (4d, $\left.4 \mathrm{H}, \mathrm{J}=12.0 \mathrm{~Hz}, \mathrm{CH}_{2} \mathrm{Ph}\right), 5.43(\mathrm{~s}, 1 \mathrm{H}, \mathrm{CHPh}), 6.95-7.08(\mathrm{~m}, 2 \mathrm{H}), 7.23-7.42(\mathrm{~m}, 20 \mathrm{H}$, aromatic), 7.50-7.58 (m, 2H); ${ }^{13} \mathrm{C}-\mathrm{NMR}\left(100 \mathrm{MHz}, \mathrm{CDCl}_{3}\right): \delta$ 21.4, 49.7, 69.0, 69.6, 70.3, 71.5, 72.7, 73.0, 73.7, 74.2, 74.7, 75.4, 82.4, 83.8, 88.4, 101.1, 106.1, 126.6, 126.9, 127.79, $127.83,127.9,128.0,128.2$, 128.4, 128.5, 128.66, 128.68, 129.1, 129.9, 134.3, 138.08, 138.14, 138.3, 138.5, 138.6. HRMS: $[\mathrm{M}+\mathrm{Na}]^{+} \mathrm{C}_{47} \mathrm{H}_{51} \mathrm{NNaO}_{9} \mathrm{~S}$ calcd 828.3182, obsd 828.3182. pTolyl 3,4,6-tri- $O$-benzyl- $\beta$-d-galactopyranosyl-( $\rightarrow 3$-4,6-benzylidene-2-amino-2-deoxy-1thio- $\beta$-р-galactopyranoside $\mathrm{S} 7(1.06 \mathrm{~g}, 1.31 \mathrm{mmol})$ and solid $\mathrm{NaHCO}_{3}(0.22 \mathrm{~g}, 2.62 \mathrm{mmol})$ were put into THF $(16 \mathrm{~mL})$ and then TrocCl $(0.214 \mathrm{~mL}, 1.57 \mathrm{mmol})$ was added. The mixture was stirred at room temperature under $\mathrm{N}_{2}$ for 4 hours and filtrated. The filtrate was concentrated and then diluted with $\mathrm{CH}_{2} \mathrm{Cl}_{2}(100 \mathrm{~mL})$. The mixture was washed with water and brine, dried over $\mathrm{Na}_{2} \mathrm{SO}_{4}$, filtered and concentrated. Silica gel column chromatography $(2: 1$ HexanesEtOAc) afforded compound 13 as white solid $(1.2 \mathrm{~g}, 93 \%)$; $[\alpha]_{\mathrm{D}}-13.6\left(c=1, \mathrm{CH}_{2} \mathrm{Cl}_{2}\right) ;{ }^{1} \mathrm{H}-$ NMR (600 MHz, $\left.\mathrm{CDCl}_{3}\right): \boldsymbol{\delta} 2.32\left(\mathrm{~s}, 3 \mathrm{H}, \mathrm{SPhCH}_{3}\right), 3.34\left(\mathrm{~d}, 1 \mathrm{H}, J_{2}{ }^{\prime}, 3^{\prime}=7.8 \mathrm{~Hz}, J_{3}{ }^{\prime}, 4^{\prime},=3.0 \mathrm{~Hz}\right.$, H-3'), 3.40 (s, 1H, H-5), 3.48-3.61 (m, 3H, H-5', H-6a', H-6b'), 3.65 (dd, 1H, J $1,2=10.2$ Hz, $\left.J_{\mathrm{NH}, 2}=7.2 \mathrm{~Hz}, \mathrm{H}-2\right), 3.82$ (d, 1H, $J_{3}{ }^{\prime}, 4^{\prime}=3.0 \mathrm{~Hz}, \mathrm{H}-4^{\prime}$ ), 3.87 (d, 1H, J = $\left.12.0 \mathrm{~Hz}, \mathrm{H}-6 \mathrm{a}\right), 3.92$ (t, $1 \mathrm{H}, J=7.8$ Hz, H-2'), 4.24-4.29 (m, 3H, H-3. H-4, H-6a), 4.35 (d, 1H, J ', , ' = 7.8 Hz, H-1'), 4.46 (s, $2 \mathrm{H}, \mathrm{CH}_{2} \mathrm{CCl}_{3}$ ), $4.58\left(\mathrm{~d}, 1 \mathrm{H}, J=12.0 \mathrm{~Hz}, \mathrm{CH}_{2} \mathrm{Ph}\right), 4.66-4.71(\mathrm{~m}, 3 \mathrm{H}, J=12.0 \mathrm{~Hz}$, 
$\left.\mathrm{CH}_{2} \mathrm{Ph}\right), 4.78,4.88\left(2 \mathrm{~d}, 2 \mathrm{H}, J=12.0 \mathrm{~Hz}, \mathrm{CH}_{2} \mathrm{Ph}\right), 5.07\left(\mathrm{~d}, 1 \mathrm{H}, J_{1,2}=10.2 \mathrm{~Hz}, \mathrm{H}-1\right), 5.46(\mathrm{~s}$, $1 \mathrm{H}, \mathrm{CHPh}), 5.52\left(\mathrm{~d}, 1 \mathrm{H}, J_{\mathrm{NH}, 2}=7.2 \mathrm{~Hz}, \mathrm{NH}\right), 7.00-7.09(\mathrm{~m}, 2 \mathrm{H}), 7.21-7.44(\mathrm{~m}, 20 \mathrm{H}$, aromatic), 7.50-7.59 (m, 2H); ${ }^{13} \mathrm{C}-\mathrm{NMR}\left(150 \mathrm{MHz}, \mathrm{CDCl}_{3}\right): \delta$ 21.5, 51.8, 69.2, 69.5, 70.3, 71.3, 73.0, 73.4, 73.8, 74.2, 74.7, 74.8, 76.1, 77.3, 81.9, 84.3, 95.9, 101.1, 105.0, 127.0, 127.4, 127.9, 128.0, 128.0, 128.1, 128.2, 128.3, 128.5, 128.7, 128.76, 128.77, 129.2, 130.0, 134.5, 138.10, 138.12, 138.4, 138.6, 154.3; HRMS: $[\mathrm{M}+\mathrm{Na}]^{+} \mathrm{C}_{50} \mathrm{H}_{52} \mathrm{Cl}_{3} \mathrm{NNaO}_{11} \mathrm{~S}$ calcd 1002.2224, obsd 1002.2208.

\section{p-Tolyl 2,4,6-tri-O-benzyl-1-thio- $\beta$-o-galactopyranoside (19)}

$\boldsymbol{\beta}$-D-Galactose pentaacetate $(20 \mathrm{~g}, 51.2 \mathrm{mmol})$ and $p$-toluenethiol $(7.3 \mathrm{~g}, 58.8 \mathrm{mmol})$ were dissolved in $\mathrm{CH}_{2} \mathrm{Cl}_{2}$. $(400 \mathrm{~mL})$. Boron trifluoride etherate $(20.15 \mathrm{~mL}, 153.6 \mathrm{mmol})$ was added dropwise at $0{ }^{\circ} \mathrm{C}$ and the mixture was stirred under $\mathrm{N}_{2}$ at room temperature for $20 \mathrm{~h}$. The mixture was diluted with $\mathrm{CH}_{2} \mathrm{Cl}_{2}(450 \mathrm{~mL})$ and washed with saturated $\mathrm{NaHCO}_{3}$, dried over $\mathrm{Na}_{2} \mathrm{SO}_{4}$, filtered and concentrated. Silica gel column chromatography (2:1 Hexanes-EtOAc) afforded $p$-tolyl 2,3,4,6-tetra- $O$-acetyl-1-thio- $\beta$-- -galactopyranoside (S8) as white solid (21.2 g, 91\%). ${ }^{1} \mathrm{H}-\mathrm{NMR}\left(600 \mathrm{MHz}, \mathrm{CDCl}_{3}\right): \boldsymbol{\delta} 1.98,2.05,2.10,2.12\left(\mathrm{~s}, 12 \mathrm{H}, 4 \times \mathrm{COCH}_{3}\right), 2.35(\mathrm{~s}$, $\left.3 \mathrm{H}, \mathrm{SPhCH}_{3}\right), 3.92$ (t, 1H, $\left.J=6.6 \mathrm{~Hz}, \mathrm{H}-5\right), 4.10-4.20$ (m, 2H, H-6a, H-6b), 4.66 (d, 1H, $\left.J_{1,2}=10.2 \mathrm{~Hz}, \mathrm{H}-1\right), 5.04\left(\mathrm{dd}, 1 \mathrm{H}, J_{2,3}=9.6 \mathrm{~Hz}, J_{3.4}=3.0 \mathrm{~Hz}, \mathrm{H}-3\right), 5.22(\mathrm{t}, 1 \mathrm{H}, J=10.2 \mathrm{~Hz}$, $\mathrm{H}-2), 5.41\left(\mathrm{~d}, 1 \mathrm{H}, J_{3.4}=3.0 \mathrm{~Hz}, \mathrm{H}-4\right), 7.08-7.16(\mathrm{~m}, 2 \mathrm{H}), 7.40-7.46(\mathrm{~m}, 2 \mathrm{H}) ;{ }^{13} \mathrm{C}-\mathrm{NMR}(150$ $\left.\mathrm{MHz}, \mathrm{CDCl}_{3}\right): \boldsymbol{\delta} 20.82,20.86,20.90,21.1,21.4,61.8,67.4,67.4,67.5,72.2,74.5,87.1,128.8$, $129.9,133.3,138.7,160.74$ 169.7, 170.3, 170.4, 170.6. ESI-MS $[\mathrm{M}+\mathrm{Na}]^{+} \mathrm{C}_{21} \mathrm{H}_{26} \mathrm{NaO}_{9} \mathrm{~S}$ calcd 477.1, obsd 477.3; Comparison of the NMR data with those reported in the literature ${ }^{63}$ confirmed its identity. $p$-Tolyl 2,3,4,6-tetra- $O$-acetyl-1-thio- $\beta$ - -galactopyranoside S8 (11.5 g, $25.3 \mathrm{mmol}$ ) was dissolved in a mixture of $\mathrm{CH}_{2} \mathrm{Cl}_{2} / \mathrm{MeOH}(100 \mathrm{~mL} / 70 \mathrm{~mL})$ and $5.14 \mathrm{M} \mathrm{NaOMe}$ $(2.4 \mathrm{~mL}, 12.7 \mathrm{mmol}$ ) was added. The mixture was stirred at room temperature for $6 \mathrm{~h}$ under $\mathrm{N}_{2}$ and then was neutralized with Amberlite IR-120 and concentrated to dryness. Silica gel column chromatography (10:1 $\left.\mathrm{CH}_{2} \mathrm{Cl}_{2}-\mathrm{MeOH}\right)$ afforded $p$-tolyl 1-thio- $\boldsymbol{\beta}$-D-galactopyranoside (S9) as white solid (7.2 g, quantitative). ${ }^{1} \mathrm{H}-\mathrm{NMR}\left(600 \mathrm{MHz}, \mathrm{CD}_{3} \mathrm{OD}\right): \boldsymbol{\delta} 2.28$ (s, $3 \mathrm{H}$, $\left.\mathrm{SPhCH}_{3}\right), 3.47\left(\mathrm{dd}, 1 \mathrm{H}, J_{2,3}=9.6 \mathrm{~Hz}, J_{3.4}=3.0 \mathrm{~Hz}, \mathrm{H}-3\right), 3.51(\mathrm{t}, 1 \mathrm{H}, J=6.6 \mathrm{~Hz}, \mathrm{H}-5), 3.55$ (t, $1 \mathrm{H}, J=9.6 \mathrm{~Hz}, \mathrm{H}-2), 3.67-3.75$ (m, 2H, H-6a, H-6b), 3.87 (d, $\left.1 \mathrm{H}, J_{3.4}=3.0 \mathrm{~Hz}, \mathrm{H}-4\right), 4.49$ $\left(\mathrm{d}, 1 \mathrm{H}, J_{1,2}=9.6 \mathrm{~Hz}, \mathrm{H}-1\right), 7.05-7.12(\mathrm{~m}, 2 \mathrm{H}), 7.40-7.47(\mathrm{~m}, 2 \mathrm{H}) ;{ }^{13} \mathrm{C}-\mathrm{NMR}(150 \mathrm{MHz}$, $\left.\mathrm{CD}_{3} \mathrm{OD}\right): \boldsymbol{\delta} 19.9,61.4,69.2,69.8,75.1,79.4,89.5,129.4,130.9,131.7,137.2$. ESI-MS [M $+\mathrm{Na}]^{+} \mathrm{C}_{13} \mathrm{H}_{18} \mathrm{NaO}_{5} \mathrm{~S}$ calcd 309.1, obsd 309.1. $p$-Tolyl 1-thio- $\boldsymbol{\beta}$-d-galactopyranoside $\mathrm{S} 9$ (3 g, $10.4 \mathrm{mmol})$ and dibutyltin oxide $(2.6 \mathrm{~g}, 10.4 \mathrm{mmol})$ were put into $\mathrm{MeOH}(45 \mathrm{~mL})$. The mixture was heated at reflux for 2 hours and then concentrated to dryness. DMF $(30 \mathrm{~mL})$ was added to the resulting residue, $p$-methoxy benzyl chloride ( $\mathrm{PMBCl}, 1.5 \mathrm{~mL}, 10.4 \mathrm{mmol})$ and $\mathrm{CsF}(1.67$ $\mathrm{g}, 10.4 \mathrm{mmol}$ ), which was then stirred under $\mathrm{N}_{2}$ at $50^{\circ} \mathrm{C}$ for 2 days and concentrated to dryness. Silica gel column chromatography (1:2 Hexanes-EtOAc) afforded $p$-tolyl 3-O-pmethoxylbenzyl-1-thio- $\boldsymbol{\beta}$-D-galactopyranoside (S10) as white solid (2.34 g, 55\% for two steps). ${ }^{1} \mathrm{H}-\mathrm{NMR}\left(600 \mathrm{MHz}, \mathrm{CD}_{3} \mathrm{OD}\right): \delta 2.20$ (s, $\left.3 \mathrm{H}, \mathrm{SPhCH}_{3}\right), 3.27$ (dd, $1 \mathrm{H}, J_{2,3}=9.6 \mathrm{~Hz}$, $\left.J_{3.4}=3.0 \mathrm{~Hz}, \mathrm{H}-3\right), 3.35-3.38$ (m, 1H, H-5), 3.58-3.65 (m, 3H, $J_{1,2}=10.2 \mathrm{~Hz}, \mathrm{H}-2, \mathrm{H}-6 \mathrm{a}$, $\mathrm{H}-6 \mathrm{~b}), 3.67$ (s, $\left.3 \mathrm{H}, \mathrm{OCH}_{3}\right), 3.94\left(\mathrm{~d}, 1 \mathrm{H}, J_{3.4}=3.0 \mathrm{~Hz}, \mathrm{H}-4\right), 4.41\left(\mathrm{~d}, 1 \mathrm{H}, J_{1,2}=10.2 \mathrm{~Hz}, \mathrm{H}-1\right)$, $4.51,4.58\left(2 \mathrm{~d}, 2 \mathrm{H}, J=11.4 \mathrm{~Hz}, \mathrm{CH}_{2} \mathrm{PhOCH}_{3}\right), 6.72-6.82(\mathrm{~m}, 2 \mathrm{H}), 6.96-7.05(\mathrm{~m}, 2 \mathrm{H}), 7.20-$ 7.28 (m, 2H), 7.30-7.38 (m, 2H); ${ }^{13} \mathrm{C}-\mathrm{NMR}\left(100 \mathrm{MHz}, \mathrm{CD}_{3} \mathrm{OD}\right): \boldsymbol{\delta} 21.3,55.76,55.81,62.7$, 67.6, 70.3, 72.5, 80.5, 83.5, 90.8, 114.8, 130.64, 130.68, 130.8, 130.9, 131.9, 132.1, 133.1, 133.2, 138.6, 160.9. ESI-MS [M+Na] ${ }^{+} \mathrm{C}_{21} \mathrm{H}_{26} \mathrm{NaO}_{6} \mathrm{~S}$ calcd 429.2, obsd 429.3. $p$-Tolyl 3-O$p$-methoxylbenzyl-1-thio- $\boldsymbol{\beta}$-D-galactopyranoside $\mathrm{S} 10(3.4 \mathrm{~g}, 8.36 \mathrm{mmol})$ was dissolved in DMF $(50 \mathrm{~mL})$ and the solution was cooled to $0{ }^{\circ} \mathrm{C}$. $\mathrm{NaH}(1.34 \mathrm{~g}, 60 \% \mathrm{NaH}$ in mineral oil, 33.44 $\mathrm{mmol}$ ) was added in portions, followed by addition of $\mathrm{BnBr}(4 \mathrm{~mL}, 33.44 \mathrm{mmol})$. The mixture was stirred at room temperature under $\mathrm{N}_{2}$ overnight and then diluted with EtOAc $(250 \mathrm{~mL})$. The mixture was washed with saturated $\mathrm{NaHCO}_{3}$, water and then dried over $\mathrm{Na}_{2} \mathrm{SO}_{4}$, filtered and concentrated. Silica gel column chromatography (8:1 Hexanes-EtOAc) afforded 
compound $14 p$-tolyl 2,4,6-tri- $O$-benzyl-3- $O$ - $p$-methoxylbenzyl-1-thio- $\boldsymbol{\beta}$-D-galactopyranoside as white solid $(4.5 \mathrm{~g}, 80 \%) ;[\alpha]_{\mathrm{D}}+3.2\left(c=1, \mathrm{CH}_{2} \mathrm{Cl}_{2}\right) ;{ }^{1} \mathrm{H}-\mathrm{NMR}\left(600 \mathrm{MHz}, \mathrm{CDCl}_{3}\right): \boldsymbol{\delta} 2.27$ (s, 3H, $\mathrm{SPhCH}_{3}$ ), 3.56-3.65 (m, 4H, H-3, H-5, H-6a, H-6b), 3.77 (s, 3H, $\mathrm{OCH}_{3}$ ), 3.87 (t, $1 \mathrm{H}$, $J=9.6 \mathrm{~Hz}, \mathrm{H}-2), 3.93\left(\mathrm{~d}, 1 \mathrm{H}, J_{3.4}=2.4 \mathrm{~Hz}, \mathrm{H}-4\right), 4.40,4.45,4.58,4.62,4.65,4.72,4.78,4.95$ $\left(8 \mathrm{~d}, 8 \mathrm{H}, J=12.0 \mathrm{~Hz}, \mathrm{CH}_{2} \mathrm{PhOCH}_{3}, \mathrm{CH}_{2} \mathrm{Ph}\right), 4.58\left(\mathrm{~d}, 1 \mathrm{H}, J_{1,2}=10.2 \mathrm{~Hz}, \mathrm{H}-1\right), 6.78-6.88(\mathrm{~m}$, 2H), 6.94-7.02 (m, 2H), 7.24-7.34 (m, 15H, aromatic), 7.35-7.45 (m, 2H), 7.40-7.49 (m, $2 \mathrm{H}) ;{ }^{13} \mathrm{C}-\mathrm{NMR}\left(150 \mathrm{MHz}, \mathrm{CDCl}_{3}\right): \boldsymbol{\delta} 21.4,55.5,69.1,72.7,73.8,73.9,74.7,75.9,77.5,84.2$, $88.3,114.1,127.7,127.96,128.04,128.1,128.2,128.4,128.57,128.59,128.68,129.5,129.8$, $130.5,130.7,132.5,137.4,138.2,138.7,139.1,159.5$. ESI-MS $[\mathrm{M}+\mathrm{Na}]^{+} \mathrm{C}_{42} \mathrm{H}_{44} \mathrm{NaO}_{6} \mathrm{~S}$ calcd 699.3, obsd 699.5.

Compound $14(0.3 \mathrm{~g}, 0.44 \mathrm{mmol})$ was dissolved in a mixture of $\mathrm{CH}_{2} \mathrm{Cl}_{2} / \mathrm{H}_{2} \mathrm{O}(2.9 \mathrm{~mL} / 0.15 \mathrm{~mL})$ and the solution was cooled to $0{ }^{\circ} \mathrm{C}$. 2,3-Dichloro 5,6-dicyano-1,4-benzoquinone (DDQ, 0.12 $\mathrm{g}, 0.53 \mathrm{mmol}$ ) was added and the mixture was stirred at room temperature for $3 \mathrm{~h}$. The mixture was filtered, diluted with $\mathrm{CH}_{2} \mathrm{Cl}_{2}(30 \mathrm{~mL})$ and the organic phase was washed with $\mathrm{H}_{2} \mathrm{O}$ until the solution became colorless. Silica gel column chromatography (4:1 Hexanes-EtOAc) afforded compound 19 as white solid $(0.21 \mathrm{~g}, 85 \%)$; [ $\alpha]_{\mathrm{D}}-3.9\left(c=3.3, \mathrm{CH}_{2} \mathrm{Cl}_{2}\right) ;{ }^{1} \mathrm{H}-\mathrm{NMR}$ $\left(600 \mathrm{MHz}, \mathrm{CDCl}_{3}\right): \delta 2.21(\mathrm{~d}, 1 \mathrm{H}, J=5.4 \mathrm{~Hz}, \mathrm{OH}), 2.30\left(\mathrm{~s}, 3 \mathrm{H}, \mathrm{SPhCH}_{3}\right), 3.63-3.68(\mathrm{~m}, 5 \mathrm{H}$, H-2, H-3, H-5, H-6a, H-6b), 3.89 (d, 1H, J $3.4=2.4 \mathrm{~Hz}, \mathrm{H}-4), 4.44,4.50(2 \mathrm{~d}, 2 \mathrm{H}, J=12.0 \mathrm{~Hz}$, $\left.\mathrm{CH}_{2} \mathrm{Ph}\right), 4.56\left(\mathrm{~d}, 1 \mathrm{H}, J_{1,2}=9.0 \mathrm{~Hz}, \mathrm{H}-1\right), 4.64\left(\mathrm{~d}, 2 \mathrm{H}, J=12.0 \mathrm{~Hz}, \mathrm{CH}_{2} \mathrm{Ph}\right), 4.73,4.90(2 \mathrm{~d}$, $\left.2 \mathrm{H}, J=12.0 \mathrm{~Hz}, \mathrm{CH}_{2} \mathrm{Ph}\right), 6.98-7.08(\mathrm{~m}, 2 \mathrm{H}), 7.28-7.37(\mathrm{~m}, 15 \mathrm{H}), 7.42-7.52(\mathrm{~m}, 2 \mathrm{H}) ;{ }^{13} \mathrm{C}-$ $\operatorname{NMR}\left(150 \mathrm{MHz}, \mathrm{CDCl}_{3}\right)$ : $\boldsymbol{\delta} 21.3,68.8,73.7,75.1,75.4,75.9,76.2,77.5,78.5,87.9,127.9$, $127.98,128.03,128.05,128.2,128.53,128.56,128.60,128.7,129.8,130.4,132.2,137.5$, 138.0, 138.3, 138.7; HRMS: [M+Na] ${ }^{+} \mathrm{C}_{34} \mathrm{H}_{36} \mathrm{NaO}_{5} \mathrm{~S}$ calcd 579.2181, obsd 579.2167.

\section{3-Azidopropyl 2,3,6-tri-O-benzyl- $\beta$-o-galactopyranosyl-(1 $\rightarrow 4)-2,3,6$-tri-O-benzyl- $\beta$-o- glucopyranoside (15)}

D-Lactose ( $(5 \mathrm{~g}, 13.87 \mathrm{mmol})$ was dissolved in pyridine $(30 \mathrm{~mL})$ and then $\mathrm{BzCl}(20 \mathrm{~mL}, 172$ mmol) was added at $0{ }^{\circ} \mathrm{C}$. The mixture was stirred at room temperature under $\mathrm{N}_{2}$ overnight and then diluted with $\mathrm{CH}_{2} \mathrm{Cl}_{2}(400 \mathrm{~mL})$. The mixture was washed with saturated $\mathrm{NaHCO}_{3}$, water and then dried over $\mathrm{Na}_{2} \mathrm{SO}_{4}$, filtered and concentrated. The resulting residue was dissolved in $\mathrm{CH}_{2} \mathrm{Cl}_{2}(30 \mathrm{~mL})$ and the solution was cooled to $-10{ }^{\circ} \mathrm{C}$. $\mathrm{HBr}$ in acetic acid (60 $\mathrm{mL}, 33 \% \mathrm{w} / \mathrm{w}, 104 \mathrm{mmol}$ ) was added and the mixture was stirred at room temperature under $\mathrm{N}_{2}$ for 5 hours. The mixture was diluted with $\mathrm{CH}_{2} \mathrm{Cl}_{2}(200 \mathrm{~mL})$ and poured into crushed ice in saturated $\mathrm{NaHCO}_{3}(400 \mathrm{~mL})$. The organic phase was separated and washed again with saturated $\mathrm{NaHCO}_{3}$ until the $\mathrm{pH}$ is about 7 and then dried over $\mathrm{Na}_{2} \mathrm{SO}_{4}$, filtered and concentrated. The resulting residue ( $2 \mathrm{~g}, 1.76 \mathrm{mmol}), 1$-bromo propanol $(2.3 \mathrm{~mL}, 26.4 \mathrm{mmol})$ and freshly activated molecular sieve MS $4 \AA(300 \mathrm{mg})$ was put into a $100 \mathrm{~mL}$ round-bottomed flask containing $\mathrm{CH}_{2} \mathrm{Cl}_{2}(20 \mathrm{~mL})$ and the mixture was stirred at room temperature for 30 minutes. AgOTf $(0.54 \mathrm{~g}, 2.11 \mathrm{mmol})$ was added and the mixture was stirred at room temperature for 1 hour. The mixture was diluted with $\mathrm{CH}_{2} \mathrm{Cl}_{2}(100 \mathrm{~mL})$ and filtered. The filtrate was washed with saturated aqueous $\mathrm{NaHCO}_{3}$ and $\mathrm{H}_{2} \mathrm{O}$. The organic layer was dried over $\mathrm{Na}_{2} \mathrm{SO}_{4}$, filtered and concentrated. Silica gel column chromatography (3:1 Hexanes-EtOAc) afforded compound 3-bromopropyl 2,3,4,6-tetra- $O$-benzoyl- $\boldsymbol{\beta}$-р-galactopyranosyl$(1 \rightarrow 4)$-2,3,6-tri- $O$-benzoyl- $\beta$-- -glucopyranoside (S11) as white solid $(1.6 \mathrm{~g}, 65 \%$ for 3 steps). ${ }^{1} \mathrm{H}-\mathrm{NMR}\left(600 \mathrm{MHz}, \mathrm{CDCl}_{3}\right): \boldsymbol{\delta} 1.96\left(\mathrm{~m}, 2 \mathrm{H}, \mathrm{OCH}_{2} \mathrm{CH}_{2} \mathrm{CH}_{2} \mathrm{~N}_{3}\right), 3.27(\mathrm{~m}, 2 \mathrm{H}$, $\mathrm{OCH}_{2} \mathrm{CH}_{2} \mathrm{CH}_{2} \mathrm{~N}_{3}$ ), 3.64 (m, $1 \mathrm{H}, \mathrm{CH}_{2} \mathrm{~N}_{3}$ ), 3.73-3.81 (m, 2H, H-5', H-6a'), 3.88-3.98 (m, 3H, H-5, H-6b', $\mathrm{CH}_{2} \mathrm{~N}_{3}$ ), 4.32 (t, $\left.1 \mathrm{H}, J=9.6 \mathrm{~Hz}, \mathrm{H}-4\right), 4.56$ (dd, $\left.1 \mathrm{H}, J=4.2,12.0 \mathrm{~Hz}, \mathrm{H}-6 \mathrm{a}\right), 4.67$ (d, $1 \mathrm{H}, J=12.0 \mathrm{~Hz}, \mathrm{H}-6 \mathrm{~b}), 4.75$ (d, 1H, $\left.J_{1,2}=7.8 \mathrm{~Hz}, \mathrm{H}-1\right), 4.96$ (d, 1H, $J_{1^{\prime}, 2^{\prime}}=7.8 \mathrm{~Hz}, \mathrm{H}-1^{\prime}$ ), $5.45\left(\mathrm{dd}, 1 \mathrm{H}, J_{2}{ }^{\prime}, 3^{\prime}=10.2 \mathrm{~Hz}, J_{3^{\prime}, 4^{\prime}}=3.0 \mathrm{~Hz}, \mathrm{H}-3^{\prime}\right), 5.51(\mathrm{t}, 1 \mathrm{H}, J=9.6 \mathrm{~Hz}, \mathrm{H}-2), 5.78-5.80$ (m, 2H, H-2', H-4'), 5.87 (t, 1H, $J=9.6 \mathrm{~Hz}, \mathrm{H}-3$ ), 7.14-8.04 (m, 35H, aromatic); ${ }^{13} \mathrm{C}-\mathrm{NMR}$ $\left(100 \mathrm{MHz}, \mathrm{CDCl}_{3}\right): \delta$ 30.1, 32.3, 61.1, 62.4, 67.5, 69.9, 71.4, 71.78, 71.82, 72.83, 73.0, 76.0, 
101.1, 101.4, 128.3, 128.4, 128.56, 128.63, 128.8, 129.2, 129.4, 129.5, 129.57, 129.62, 129.68, 129.74, 130.0, 133.2, 133.3, 133.4, 133.6, 164.8, 165.2, 165.3, 165.4, 165.5, 165.8. ESI-MS $[\mathrm{M}+\mathrm{Na}]^{+} \mathrm{C}_{64} \mathrm{H}_{55} \mathrm{NaBrO}_{18}$ calcd 1213.3, obsd 1213.6. 3-Bromopropyl 2,3,4,6-tetra-Obenzoyl- $\boldsymbol{\beta}$-D-galactopyranosyl-( $1 \rightarrow 4)$-2,3,6-tri- $O$-benzoyl- $\boldsymbol{\beta}$-D-glucopyranoside S11 $(1.2 \mathrm{~g}, 1$ $\mathrm{mmol})$ and $\mathrm{NaN}_{3}(0.66 \mathrm{~g}, 10 \mathrm{mmol})$ were dissolved in DMF $(10 \mathrm{~mL})$. The mixture was stirred at $60{ }^{\circ} \mathrm{C}$ for $30 \mathrm{~h}$ and then concentrated. The resulting residue was diluted with EtOAc (200 $\mathrm{mL}$ ), washed with $\mathrm{H}_{2} \mathrm{O}$ and then dried over $\mathrm{Na}_{2} \mathrm{SO}_{4}$, filtered and concentrated. Silica gel column chromatography (3:1 Hexanes-EtOAc) afforded compound 3-azidopropyl 2,3,4,6tetra- $O$-benzoyl- $\boldsymbol{\beta}$ - - -galactopyranosyl-( $1 \rightarrow 4$ )-2,3,6-tri- $O$-benzoyl- $\boldsymbol{\beta}$-р-glucopyranoside (S12) as white solid (1.04 g, 90\%). ${ }^{1} \mathrm{H}-\mathrm{NMR}\left(600 \mathrm{MHz}, \mathrm{CDCl}_{3}\right)$ : $\boldsymbol{\delta} 1.72\left(\mathrm{~m}, 2 \mathrm{H}, \mathrm{CH}_{2} \mathrm{CH}_{2} \mathrm{CH}_{2} \mathrm{~N}_{3}\right)$, $3.18\left(\mathrm{~m}, 2 \mathrm{H}, \mathrm{OCH}_{2} \mathrm{CH}_{2} \mathrm{CH}_{2} \mathrm{~N}_{3}\right), 3.54\left(\mathrm{~m}, 1 \mathrm{H}, \mathrm{CH}_{2} \mathrm{~N}_{3}\right), 3.71-3.78$ (m, 2H, H-5', H-6a'), 3.863.95 (m, 3H, H-5, H-6b', $\left.\mathrm{CH}_{2} \mathrm{~N}_{3}\right), 4.29$ (t, $\left.1 \mathrm{H}, J=9.0 \mathrm{~Hz}, \mathrm{H}-4\right), 4.53$ (dd, $1 \mathrm{H}, J=4.2,12.0$ $\mathrm{Hz}, \mathrm{H}-6 \mathrm{a}), 4.64$ (d, 1H, J = 12.0 Hz, H-6b), 4.71 (d, 1H, $\left.J_{1,2}=7.8 \mathrm{~Hz}, \mathrm{H}-1\right), 4.93$ (d, 1H, $\left.J_{1^{\prime}, 2^{\prime}}=7.8 \mathrm{~Hz}, \mathrm{H}^{\prime} 1^{\prime}\right), 5.43\left(\mathrm{dd}, 1 \mathrm{H}, J_{2^{\prime}, 3^{\prime}}=10.2 \mathrm{~Hz}, J_{3^{\prime}, 4^{\prime}}=3.0 \mathrm{~Hz}, \mathrm{H}-3^{\prime}\right), 5.49(\mathrm{t}, 1 \mathrm{H}, J=7.8$ Hz, H-2), 5.75-5.78 (m, 2H, H-2', H-4'), 5.85 (t, 1H, J = 9.6 Hz, H-3), 7.14-8.12 (m, 35H, aromatic); ${ }^{13} \mathrm{C}-\mathrm{NMR}\left(100 \mathrm{MHz}, \mathrm{CDCl}_{3}\right): \delta$ 29.1, 48.0, 61.2, 62.5, 66.8, 67.7, 70.0, 71.5, 71.9, 72.0, 73.0, 73.2, 76.2, 101.2, 101.4, 128.4, 128.67, 128.71, 128.76, 128.82, 128.99, 129.4, 129.6, 129.66, 129.74, 129.77, 129.84, 129.86, 129.93, 130.18, 130.37, 133.40, 133.5, 133.6, $133.8,133.9,165.0,165.4,165.58,165.60,165.8,166.0$. ESI-MS [M+Na] ${ }^{+}$

$\mathrm{C}_{64} \mathrm{H}_{55} \mathrm{NaN}_{3} \mathrm{O}_{18}$ calcd 1176.4, obsd 1176.8. 3-Azidopropyl 2,3,4,6-tetra- $O$-benzoyl- $\boldsymbol{\beta}$-рgalactopyranosyl-( $1 \rightarrow 4)$-2,3,6-tri- $O$-benzoyl- $\beta$-- -glucopyranoside $\mathrm{S} 12(1 \mathrm{~g}, 0.86 \mathrm{mmol})$ was dissolved in $\mathrm{MeOH}(16 \mathrm{~mL})$ and $5.14 \mathrm{M} \mathrm{NaOMe}(1.67 \mathrm{~mL}, 8.6 \mathrm{mmol})$ was added. The mixture was heated at reflux for 6 hours under $\mathrm{N}_{2}$ and then was neutralized with Amberlite IR-120 until $\mathrm{pH}$ is around 7. It was filtered and concentrated to dryness. Silica gel column chromatography (4:1 $\left.\mathrm{CH}_{2} \mathrm{Cl}_{2}-\mathrm{MeOH}\right)$ afforded 3-azidopropyl $\boldsymbol{\beta}$-р-galactopyranosyl-(1 $\left.\rightarrow 4\right)-\boldsymbol{\beta}$-р-

glucopyranoside (S13) as white solid (0.36 g, 97\%). ${ }^{1} \mathrm{H}-\mathrm{NMR}\left(600 \mathrm{MHz}, \mathrm{CD}_{3} \mathrm{OD}\right): \boldsymbol{\delta} 1.85$ (m, $2 \mathrm{H}, \mathrm{CH}_{2} \mathrm{CH}_{2} \mathrm{CH}_{2} \mathrm{~N}_{3}$ ), 3.23 (t, $1 \mathrm{H}, J=7.8 \mathrm{~Hz}, \mathrm{H}-2$ '), 3.38-3.40 (m, 1H, H-5), 3.40-3.58 (m, 7H, H-2, H-3, H-6a, H-3', H-5', OCH $\left.\mathrm{CH}_{2} \mathrm{CH}_{2} \mathrm{~N}_{3}\right), 3.60-3.64\left(\mathrm{~m}, 1 \mathrm{H}, \mathrm{CH}_{2} \mathrm{~N}_{3}\right), 3.68$ (dd, $1 \mathrm{H}$, $J=4.8,12.0 \mathrm{~Hz}, \mathrm{H}-6 \mathrm{a}$ '), 3.76 (dd, $1 \mathrm{H}, J=7.8,11.4 \mathrm{~Hz}, \mathrm{H}-4), 3.80$ (d, $1 \mathrm{H}, J_{3^{\prime}, 4^{\prime}}=3.0 \mathrm{~Hz}, \mathrm{H}-4^{\prime}$ ), 3.83 (dd, $1 \mathrm{H}, J=3.6,12.0 \mathrm{~Hz}, \mathrm{H}-6 \mathrm{~b}$ '), 3.88 (dd, $1 \mathrm{H}, J=4.8,12.0 \mathrm{~Hz}, \mathrm{H}-6 \mathrm{~b}$ ), 3.92-3.96 (m, $1 \mathrm{H}, \mathrm{CH}_{2} \mathrm{~N}_{3}$ ), $4.27\left(\mathrm{~d}, 1 \mathrm{H}, J_{1^{\prime}, 2^{\prime}}=7.8 \mathrm{~Hz}, \mathrm{H}-1^{\prime}\right), 4.34\left(\mathrm{~d}, 1 \mathrm{H}, J_{1,2}=7.8 \mathrm{~Hz}, \mathrm{H}-1\right) ;{ }^{13} \mathrm{C}-\mathrm{NMR}$ $\left(150 \mathrm{MHz}, \mathrm{CD}_{3} \mathrm{OD}\right): \boldsymbol{\delta} 29.0,48.2,60.7,61.3,66.4,69.1,71.4,73.5,73.6,75.2,75.3,75.9$, 79.4, 103.1, 103.9. ESI-MS $[\mathrm{M}+\mathrm{Na}]^{+} \mathrm{C}_{15} \mathrm{H}_{27} \mathrm{NaN}_{3} \mathrm{O}_{11}$ calcd 448.2, obsd 448.2. The mixture of 3-azidopropyl $\boldsymbol{\beta}$-р-galactopyranosyl-(1 $\rightarrow 4)$ - $\boldsymbol{\beta}$-р-glucopyranoside $\mathrm{S} 13(0.5 \mathrm{~g}, 1.17 \mathrm{mmol})$, camphorsulfonic acid $(0.14 \mathrm{~g}, 0.59 \mathrm{mmol})$, benzaldehyde dimethylacetal $(0.2 \mathrm{~mL}, 1.35 \mathrm{mmol})$ and DMF $(3 \mathrm{~mL})$ was stirred at room temperature under $\mathrm{N}_{2}$ overnight. The mixture was neutralized with solid $\mathrm{NaHCO}_{3}(0.98 \mathrm{~g}, 1.17 \mathrm{mmol})$ and then concentrated to dryness. Silica gel column chromatography $\left(8: 1 \mathrm{CH}_{2} \mathrm{Cl}_{2}-\mathrm{MeOH}\right)$ afforded 3-azidopropyl 4,6-O-benzylidene$\boldsymbol{\beta}$-D-galactopyranosyl- $(1 \rightarrow 4)$ - $\boldsymbol{\beta}$-D-glucopyranoside ${ }^{64}(\mathrm{~S} 14)$ as white solid $(0.49 \mathrm{~g}, 81 \%) .{ }^{1} \mathrm{H}$ NMR (600 MHz, CD ${ }_{3} \mathrm{OD}$ ): $\boldsymbol{\delta} 1.80$ (m, 2H, $\mathrm{CH}_{2} \mathrm{CH}_{2} \mathrm{CH}_{2} \mathrm{~N}_{3}$ ), 3.21 (t, $1 \mathrm{H}, J=7.8 \mathrm{~Hz}, \mathrm{H}-2^{\prime}$ ), 3.34-3.39 (m, 3H, H-5, OCH $\mathrm{CH}_{2} \mathrm{CH}_{2} \mathrm{~N}_{3}$ ), 3.49-3.62 (m, 6H, H-2, H-3, H-6a, H-3', H-5', $\left.\mathrm{CH}_{2} \mathrm{~N}_{3}\right), 3.84-3.89\left(\mathrm{~m}, 3 \mathrm{H}, \mathrm{H}-4, \mathrm{H}-6 \mathrm{~b}, \mathrm{CH}_{2} \mathrm{~N}_{3}\right), 4.05(\mathrm{~d}, 1 \mathrm{H}, \mathrm{J}=11.4 \mathrm{~Hz}, \mathrm{H}-6 \mathrm{a}), 4.11-4.13$ (m, 2H, H-4', H-6b'), 4.21 (d, 1H, $J_{1}^{\prime}, 2^{\prime}=7.8 \mathrm{~Hz}, \mathrm{H}-1^{\prime}$ ), 4.40 (d, 1H, $J_{1,2}=7.8 \mathrm{~Hz}, \mathrm{H}-1$ ), 5.52 (s, 1H, CHPh), 7.27-7.48 (m, 5H, aromatic); ${ }^{13} \mathrm{C}-\mathrm{NMR}$ (100 MHz, $\mathrm{CD}_{3} \mathrm{OD}$ ): $\boldsymbol{\delta} 30.3,49.4$, 61.8, 67.7, 68.29, 68.33, 70.3, 71.8, 73.5, 74.8, 76.3, 76.5, 77.3, 80.1, 102.2, 104.4, 104.9, 127.6, 129.2, 130.0, 139.6. ESI-MS $[\mathrm{M}+\mathrm{Na}]^{+} \mathrm{C}_{22} \mathrm{H}_{31} \mathrm{NaN}_{3} \mathrm{O}_{11}$ calcd 536.2, obsd 536.3. 3Azidopropyl 4,6- $O$-benzylidene- $\boldsymbol{\beta}$-D-galactopyranosyl-( $1 \rightarrow 4)$ - $\boldsymbol{\beta}$-D-glucopyranoside $\mathrm{S} 14$ (2 g, $3.89 \mathrm{mmol})$ was dissolved in DMF $(25 \mathrm{~mL})$ and the solution was cooled to $0{ }^{\circ} \mathrm{C} . \mathrm{NaH}(0.93 \mathrm{~g}$, $60 \% \mathrm{NaH}$ in mineral oil, $23.34 \mathrm{mmol}$ ) was added in portions, followed by addition of $\mathrm{BnBr}$ $(2.8 \mathrm{~mL}, 33.44 \mathrm{mmol})$. The mixture was stirred at room temperature under $\mathrm{N}_{2}$ for $6 \mathrm{~h}$ and then diluted with EtOAc $(250 \mathrm{~mL})$. The mixture was washed with saturated aqueous $\mathrm{NaHCO}_{3}$, water and then dried over $\mathrm{Na}_{2} \mathrm{SO}_{4}$, filtered and concentrated. Silica gel column chromatography (6:1 Hexanes-EtOAc) afforded 3-azidopropyl 2,3-di-O-benzyl-4,6-O-benzylidene- $\boldsymbol{\beta}$-р- 
galactopyranosyl-( $1 \rightarrow 4)$-2,3,6-tri- $O$-benzyl- $\beta$-d-glucopyranoside ${ }^{53}(\mathrm{~S} 15)$ as white solid (3 g, $80 \%) ;[\alpha]_{\mathrm{D}}+176.4\left(c, 0.56, \mathrm{CH}_{2} \mathrm{Cl}_{2}\right) ;[\alpha]_{\mathrm{D}}+16.1\left(c=1, \mathrm{CHCl}_{3}\right) ;{ }^{1} \mathrm{H}-\mathrm{NMR}(600 \mathrm{MHz}$, $\left.\mathrm{CDCl}_{3}\right): \boldsymbol{\delta} 1.88\left(\mathrm{~m}, 2 \mathrm{H}, \mathrm{CH}_{2} \mathrm{CH}_{2} \mathrm{CH}_{2} \mathrm{~N}_{3}\right), 2.93$ (s, $1 \mathrm{H}, \mathrm{H}-5$ '), 3.33-3.39 (m, 4H, H-3, H-5, $\mathrm{OCH}_{2} \mathrm{CH}_{2} \mathrm{CH}_{2} \mathrm{~N}_{3}$ ), 3.42 (t, $\left.1 \mathrm{H}, J=7.8 \mathrm{~Hz}, \mathrm{H}-2^{\prime}\right), 3.60-3.64\left(\mathrm{~m}, 2 \mathrm{H}, \mathrm{H}-3^{\prime}, \mathrm{CH}_{2} \mathrm{~N}_{3}\right), 3.68-3.70$ $(\mathrm{d}, 1 \mathrm{H}, J=10.8 \mathrm{~Hz}, \mathrm{H}-6 \mathrm{a}), 3.74-3.77$ (t, $1 \mathrm{H}, J=7.8 \mathrm{~Hz}, \mathrm{H}-2), 3.84$ (d, $1 \mathrm{H}, J_{3,4}=12.0 \mathrm{~Hz}$, H-4), 3.86-3.89 (dd, 1H, $\left.J_{5}{ }^{\prime}, 6 \mathrm{a}^{\prime}=4.2 \mathrm{~Hz}, J_{6 \mathrm{a}^{\prime}, 6 \mathrm{~b}^{\prime}}=10.8 \mathrm{~Hz}, \mathrm{H}-6 \mathrm{a}^{\prime}\right), 3.96-3.98$ (m, 2H, H-6b, $\left.\mathrm{CH}_{2} \mathrm{~N}_{3}\right), 4.02\left(\mathrm{~d}, 1 \mathrm{H}, J_{3}, 4^{\prime}=3.0 \mathrm{~Hz}, \mathrm{H}-4^{\prime}\right), 4.20\left(\mathrm{~d}, 1 \mathrm{H}, J=12.0 \mathrm{~Hz}, \mathrm{H}-6 \mathrm{~b}^{\prime}\right), 4.32$, (d, $1 \mathrm{H}, J$ $\left.=12.0 \mathrm{~Hz}, \mathrm{CH}_{2} \mathrm{Ph}\right), 4.36\left(\mathrm{~d}, 1 \mathrm{H}, J_{1^{\prime}, 2^{\prime}}=7.8 \mathrm{~Hz}, \mathrm{H}-1^{\prime}\right), 4.44\left(\mathrm{~d}, 1 \mathrm{H}, J_{1,2}=7.8 \mathrm{~Hz}, \mathrm{H}-1\right), 4.54$ $\left(\mathrm{d}, 1 \mathrm{H}, J=12.0 \mathrm{~Hz}, \mathrm{CH}_{2} \mathrm{Ph}\right), 4.72-4.85\left(\mathrm{~m}, 7 \mathrm{H}, \mathrm{CH}_{2} \mathrm{Ph}\right), 5.19,5.46(2 \mathrm{~d}, 2 \mathrm{H}, J=12.0 \mathrm{~Hz}$, $\left.\mathrm{CH}_{2} \mathrm{Ph}\right), 7.17-7.52\left(\mathrm{~m}, 30 \mathrm{H}\right.$, aromatic); ${ }^{13} \mathrm{C}-\mathrm{NMR}\left(150 \mathrm{MHz}, \mathrm{CDCl}_{3}\right): \boldsymbol{\delta} 29.47,48.56,66.57$, $66.59,66.78,68.44,69.19,71.85,73.22,73.88,75.33,75.35,75.34,76.07,77.80,79.05,79.86$, 82.06, 83.30, 101.61, 103.09, 103.80, 126.81, 127.55, 127.66, 127.70, 127.87, 127.95, 127.99, $128.15,128.33,128.38,128.45,128.48,128.59,128.61,128.87,129.10,138.32,138.65$, 138.72, 138.84, 139.09, 139.13. ESI-MS $[\mathrm{M}+\mathrm{Na}]^{+} \mathrm{C}_{57} \mathrm{H}_{61} \mathrm{NaN}_{3} \mathrm{O}_{11}$ calcd 986.4, obsd 986.7. 3-Azidopropyl 2,3-di- $O$-benzyl-4,6- $O$-benzylidene- $\boldsymbol{\beta}$-ь-galactopyranosyl-(1 $\rightarrow 4$ )-2,3,6-tri- $O$ benzyl- $\beta$-D-glucopyranoside $\mathrm{S} 15(0.6 \mathrm{~g}, 0.62 \mathrm{mmol})$ and $\mathrm{NaBH}_{3} \mathrm{CN}(0.35 \mathrm{~g}, 5.58 \mathrm{mmol})$ were dissolved in THF $(15 \mathrm{~mL})$ and cooled to $0{ }^{\circ} \mathrm{C}$. A solution of $\mathrm{HCl}$ in ether $(2 \mathrm{M}, 3 \mathrm{~mL})$ was added and the mixture was stirred at room temperature for 3 hours and then concentrated to dryness. The obtained residue was diluted with $\mathrm{CH}_{2} \mathrm{Cl}_{2}$ and washed with $10 \% \mathrm{HCl}$, water and then dried over $\mathrm{Na}_{2} \mathrm{SO}_{4}$, filtered and concentrated. Silica gel column chromatography (3:1 Hexanes-EtOAc) afforded compound $\mathbf{1 5}$ as white solid $(0.53 \mathrm{~g}, 89 \%) ;[\alpha]_{\mathrm{D}}+16.1(c=1$, $\left.\mathrm{CHCl}_{3}\right) ;{ }^{1} \mathrm{H}-\mathrm{NMR}\left(600 \mathrm{MHz}, \mathrm{CDCl}_{3}\right): \boldsymbol{\delta} 1.86\left(\mathrm{~m}, 2 \mathrm{H}, \mathrm{CH}_{2} \mathrm{CH}_{2} \mathrm{CH}_{2} \mathrm{~N}_{3}\right), 2.48(\mathrm{~s}, 1 \mathrm{H}, \mathrm{OH})$, 3.30-3.41 (m, 6H, H-2', H-3, H-5, H-5', OCH $\mathrm{CH}_{2} \mathrm{CH}_{2} \mathrm{~N}_{3}$ ), 3.46-3.49 (dd, $1 \mathrm{H}, J_{5,6 \mathrm{a}}=5.4$ $\left.\mathrm{Hz}, J_{6 \mathrm{a}, 6 \mathrm{~b}}=9.6 \mathrm{~Hz}, \mathrm{H}-6 \mathrm{a}\right), 3.55-3.70\left(\mathrm{~m}, 5 \mathrm{H}, \mathrm{H}-2, \mathrm{H}-3\right.$ ', H-6a', H-6b, $\left.\mathrm{CH}_{2} \mathrm{~N}_{3}\right), 3.79-3.81$ (dd, $\left.1 \mathrm{H}, J_{5}, 6 \mathrm{a}^{\prime}=4.2 \mathrm{~Hz}, J_{6 \mathrm{a}^{\prime}, 6 \mathrm{~b}^{\prime}}=10.8 \mathrm{~Hz}, \mathrm{H}-6 \mathrm{~b}^{\prime}\right), 3.94-4.01\left(\mathrm{~m}, 3 \mathrm{H}, \mathrm{H}-4, \mathrm{H}-4^{\prime}, \mathrm{CH}_{2} \mathrm{~N}_{3}\right), 4.34-$ $4.45\left(\mathrm{~m}, 5 \mathrm{H}, J_{1,2}=7.8 \mathrm{~Hz}, J_{1}{ }^{\prime}, 2^{\prime}=8.4 \mathrm{~Hz}, \mathrm{H}-1, \mathrm{H}-1^{\prime}, \mathrm{CH}_{2} \mathrm{Ph}\right), 4.54,4.64,4.69$ (3d, 3H, $J=$ $\left.12.0 \mathrm{~Hz}, \mathrm{CH}_{2} \mathrm{Ph}\right), 4.73-4.79\left(\mathrm{~m}, 4 \mathrm{H}, \mathrm{CH}_{2} \mathrm{Ph}\right), 4.83,4.99\left(2 \mathrm{~d}, 2 \mathrm{H}, J=12.0 \mathrm{~Hz}, \mathrm{CH}_{2} \mathrm{Ph}\right), 7.19-$ 7.39 (m, 30H, aromatic); ${ }^{13} \mathrm{CNMR}\left(150 \mathrm{MHz}, \mathrm{CDCl}_{3}\right): \boldsymbol{\delta} 29.6,48.6,66.4,66.8,68.5,68.8$, 72.3, 73.1, 73.4, 73.8, 75.36, 75.39, 75.6, 75.7, 76.8, 79.7, 81.4, 82.1, 83.2, 89.5, 102.9, 103.9, $127.6,127.85,127.88,127.95,127.99,128.09,128.12,128.19,128.26,128.40,128.45,128.61$, $128.67,128.70,128.80,138.2,138.5,138.6,138.88,138.94,139.4$. ESI-MS $[\mathrm{M}+\mathrm{Na}]^{+}$ $\mathrm{C}_{57} \mathrm{H}_{63} \mathrm{NaN}_{3} \mathrm{O}_{11}$ calcd 988.5 , obsd 988.8 ;

\section{3-Azidopropyl 2,3-di-O-benzoyl-6-O-benzyl- $\beta$-o-galactopyranosyl-(1 $\rightarrow 4)-2,3,6$-tri-O-benzoyl- $\beta$-o-glucopyranoside(21)}

3-Azidopropyl 4,6-O-benzylidene- $\boldsymbol{\beta}$-о-galactopyranosyl-( $1 \rightarrow 4)$ - $\boldsymbol{\beta}$-р-glucopyranoside $(0.5 \mathrm{~g}$, $0.97 \mathrm{mmol})$ was dissolved in pyridine $(20 \mathrm{~mL})$ and then $\mathrm{BzCl}(1.1 \mathrm{~mL}, 9.7 \mathrm{mmol})$ was added at $0{ }^{\circ} \mathrm{C}$. The mixture was stirred at room temperature under $\mathrm{N}_{2}$ overnight and then diluted with $\mathrm{CH}_{2} \mathrm{Cl}_{2}(100 \mathrm{~mL})$. The mixture was washed with saturated aqueous solution of $\mathrm{NaHCO}_{3}$, water and then dried over $\mathrm{Na}_{2} \mathrm{SO}_{4}$, filtered and concentrated. The mixture of the resulting residue and $\mathrm{NaBH}_{3} \mathrm{CN}(0.6 \mathrm{~g}, 9.7 \mathrm{mmol})$ in $\mathrm{THF}(20 \mathrm{~mL})$ was cooled to $0{ }^{\circ} \mathrm{C}$ and then a solution of $\mathrm{HCl}$ in ether (2 M) was added until the solution was acidic. The mixture was stirred at room temperature for 3 hours and then concentrated to dryness. The resulting residue was diluted with $\mathrm{CH}_{2} \mathrm{Cl}_{2}$ and washed with $10 \%$ aqueous $\mathrm{HCl}$, water and then dried over $\mathrm{Na}_{2} \mathrm{SO}_{4}$, filtered and concentrated. Silica gel column chromatography (2:1 Hexanes-EtOAc) afforded compound 21 as gel-like solid $(0.7 \mathrm{~g}, 70 \%$ for two steps $) ;[\alpha]_{\mathrm{D}}+47.9\left(c=1, \mathrm{CH}_{2} \mathrm{Cl}_{2}\right) ;{ }^{1} \mathrm{H}-\mathrm{NMR}$ (600 MHz, $\mathrm{CDCl}_{3}$ ): $\boldsymbol{\delta} 1.70$ (m, 2H, $\mathrm{CH}_{2} \mathrm{CH}_{2} \mathrm{CH}_{2} \mathrm{~N}_{3}$ ), 3.01-3.08 (m, 2H, H-6a', H-6b'), 3.12$3.19\left(\mathrm{~m}, 2 \mathrm{H}, \mathrm{OCH}_{2} \mathrm{CH}_{2} \mathrm{CH}_{2} \mathrm{~N}_{3}\right), 3.45\left(\mathrm{t}, 1 \mathrm{H}, J=6.0 \mathrm{~Hz}, \mathrm{H}-5\right.$ '), 3.48-3.52 (m, $\left.1 \mathrm{H}, \mathrm{CH}_{2} \mathrm{~N}_{3}\right)$, 3.81-3.87 (m, 2H, H-5, $\mathrm{CH}_{2} \mathrm{~N}_{3}$ ), 4.17-4.26 (m, 4H, H-4, H-4', $\mathrm{CH}_{2} \mathrm{Ph}$ ), 4.42 (dd, $1 \mathrm{H}, J=4.8$, $12.0 \mathrm{~Hz}, \mathrm{H}-6 \mathrm{a}), 4.60$ (dd, $1 \mathrm{H}, J=1.8,12.0 \mathrm{~Hz}, \mathrm{H}-6 \mathrm{~b}), 4.65$ (d, $\left.1 \mathrm{H}, J_{1,2}=7.8 \mathrm{~Hz}, \mathrm{H}-1\right), 4.76$ $\left(\mathrm{d}, 1 \mathrm{H}, J_{1^{\prime}, 2^{\prime}}=7.8 \mathrm{~Hz}, \mathrm{H}^{\prime} 1^{\prime}\right), 5.12\left(\mathrm{dd}, 1 \mathrm{H}, J_{2^{\prime}, 3^{\prime}}=10.5 \mathrm{~Hz}, J_{3^{\prime}, 4^{\prime}}=3.0 \mathrm{~Hz}, \mathrm{H}-3^{\prime}\right), 5.37(\mathrm{t}, 1 \mathrm{H}$, $J=7.8 \mathrm{~Hz}, \mathrm{H}-2), 5.69-5.75$ (m, 2H, H-3, H-2'), 7.20-8.02 (m, 30H, aromatic); ${ }^{13} \mathrm{C}-\mathrm{NMR}(100$ 
$\left.\mathrm{MHz}, \mathrm{CDCl}_{3}\right): \boldsymbol{\delta} 29.0,48.0,62.5,66.6,67.2,67.5,70.1,72.0,73.1,73.1,73.4,73.5,74.4,76.4$, 101.0, 101.4, 127.7, 128.0, 128.6, 129.0, 129.2, 129.3, 129.7, 129.81, 129.84, 129.91, 129.93, 129.98, 130.01, 133.29, 133.37, 133.47, 137.8, 165.2, 165.43, 165.45, 165.88, 165.93; HRMS: $[\mathrm{M}+\mathrm{Na}]^{+} \mathrm{C}_{57} \mathrm{H}_{53} \mathrm{~N}_{3} \mathrm{NaO}_{16}$ calcd 1058.3324, obsd 1058.3315.

\section{3-Azidopropyl 2,4,6-tri-O-benzyl-3-O-p-methoxybenzyl-o-o-galactopyranosyl-(1 $\rightarrow$ 4)-2,3,6-tri- O-benzyl- $\beta$-o-galactopyranosyl-(1 $\rightarrow 4)-2,3,6$-tri-O-benzyl- $\beta$-o-glucopyranoside (16)}

After the donor 14 (400 mg, $0.59 \mathrm{mmol})$, acceptor $15(540 \mathrm{mg}, 0.56 \mathrm{mmol})$ and activated molecular sieve MS-4 $\AA$ ( $500 \mathrm{mg}$ ) were stirred for $30 \mathrm{~min}$ at room temperature in a mixture solvent of $\mathrm{Et}_{2} \mathrm{O}(8 \mathrm{~mL})$ and $\mathrm{CH}_{2} \mathrm{Cl}_{2}(16 \mathrm{~mL})$, the mixture was cooled to $-78^{\circ} \mathrm{C}$, followed by addition of AgOTf ( $456 \mathrm{mg}, 1.77 \mathrm{mmol})$ in $\mathrm{Et}_{2} \mathrm{O}(12 \mathrm{~mL})$. The mixture was vigorously stirred for $10 \mathrm{~min}$ and then $p$-TolSCl $(93.7 \mu \mathrm{L}, 0.59 \mathrm{mmol})$ was added and the reaction mixture was stirred for $2 \mathrm{~h}$ from -78 to $-40{ }^{\circ} \mathrm{C}$. (See the general procedure for single step pre-activation based glycosylation for precautions) The mixture was concentrated under vacuum to dryness. The resulting residue was diluted with $\mathrm{CH}_{2} \mathrm{Cl}_{2}(100 \mathrm{~mL})$, followed by filtration. The organic phase was washed with saturated aqueous $\mathrm{NaHCO}_{3}$ and $\mathrm{H}_{2} \mathrm{O}$ and then dried over $\mathrm{Na}_{2} \mathrm{SO}_{4}$, filtered and concentrated. Silica gel column chromatography (2:1 Hexanes-EtOAc) afforded 16 as gel-like solid $(702 \mathrm{mg}, 82 \%)$; $[\alpha]_{\mathrm{D}}+37.3\left(c=2.6, \mathrm{CH}_{2} \mathrm{Cl}_{2}\right) ;{ }^{1} \mathrm{H}-\mathrm{NMR}(600 \mathrm{MHz}$, $\left.\mathrm{CDCl}_{3}\right): \boldsymbol{\delta} 1.86\left(\mathrm{~m}, 2 \mathrm{H}, \mathrm{OCH}_{2} \mathrm{CH}_{2} \mathrm{CH}_{2} \mathrm{~N}_{3}\right), 3.15(\mathrm{dd}, 1 \mathrm{H}, J=4.8,8.4 \mathrm{~Hz}, \mathrm{H}-6 \mathrm{a}), 3.28-3.38$ (m, 6H, H-2', H-3, H-5, H-5', OCH $\mathrm{CH}_{2} \mathrm{CH}_{2} \mathrm{~N}_{3}$ ), 3.47-3.50 (m, 2H, H-5', H-6b), 3.55-3.70 (m, 4H, H-2, H-3', H-6a', $\mathrm{CH}_{2} \mathrm{~N}_{3}$ ), 3.76 (s, 3H, $\mathrm{OCH}_{3}$ ), 3.82 (m, 1H, H-6a"), 3.93-3.98 (m, $3 \mathrm{H}, \mathrm{H}-4, \mathrm{H}-4$ ', $\mathrm{CH}_{2} \mathrm{~N}_{3}$ ), 4.02-4.07 (m, 5H, H-2", H-3", H-6b', $\mathrm{CH}_{2} \mathrm{Ph}$ ), 4.17 (dd, 1H, H-4"), $4.21-4.28\left(2 \mathrm{~d}, 2 \mathrm{H}, J=12.0 \mathrm{~Hz}, \mathrm{CH}_{2} \mathrm{Ph}\right), 4.33-4.36$ (m, 4H, $J_{1^{\prime}, 2^{\prime}}=7.8 \mathrm{~Hz}, \mathrm{H}-1^{\prime}, \mathrm{H}-6 \mathrm{~b}$ ", $\left.\mathrm{CH}_{2} \mathrm{Ph}\right), 4.44-4.52\left(\mathrm{~m}, 5 \mathrm{H}, J_{1,2}=7.8 \mathrm{~Hz}, \mathrm{H}-1, \mathrm{CH}_{2} \mathrm{Ph}\right), 4.68-4.80$ (m, 7H, $\left.\mathrm{CH}_{2} \mathrm{Ph}\right), 4.85-4.87$ $\left(\mathrm{m}, 2 \mathrm{H}, \mathrm{CH}_{2} \mathrm{Ph}\right), 5.04\left(\mathrm{~d}, 1 \mathrm{H}, J_{1}\right.$ ", $2^{\prime}=3.0 \mathrm{~Hz}, \mathrm{H}-1$ "), $5.07\left(\mathrm{~d}, 1 \mathrm{H}, \mathrm{CH}_{2} \mathrm{Ph}\right), 6.77-7.46(\mathrm{~m}, 49 \mathrm{H}$, aromatic); ${ }^{13} \mathrm{C}-\mathrm{NMR}\left(150 \mathrm{MHz}, \mathrm{CDCl}_{3}\right): \boldsymbol{\delta} 29.5,48.6,55.5,66.7,67.9,68.1,68.4,69.7,72.3$, 72.4, 73.26, 73.30, 73.4, 73.50, 74.0, 75.0, 75.13, 75.19, 75.3, 75.50, 75.52, 76.7, 77.4, 79.5, $79.7,81.9,82.9,101.1,103.1,103.7,113.8,127.62,127.66,127.68,127.72,127.74,127.76$, $127.83,127.84,127.86,127.89,127.91,128.11,128.21,128.37,128.40,128.42,128.48$, $128.50,128.51,128.52,128.54,128.56,128.63,128.80,129.08,138.26,138.59,138.66$, 138.83, 138.86, 138.99, 139.01, 139.20, 139.33, 159.1; HRMS: $[\mathrm{M}+\mathrm{Na}]^{+} \mathrm{C}_{92} \mathrm{H}_{99} \mathrm{~N}_{3} \mathrm{NaO}_{17}$ calcd 1540.6872, obsd 1540.6831. gHMQC (without ${ }^{1} \mathrm{H}$ decoupling): ${ }^{1} \mathrm{~J}_{\mathrm{Cl}}{ }^{\prime}, \mathrm{H} 1{ }^{\prime}=169.0$ $\mathrm{Hz},{ }^{1} \mathrm{~J}_{\mathrm{Cl} 1}, \mathrm{H} 1,=160.1 \mathrm{~Hz},{ }^{1} \mathrm{~J}_{\mathrm{C} 1, \mathrm{H} 1}=160.0 \mathrm{~Hz}$.

\section{3-Azidopropyl 2,4,6-tri-O-benzyl- $\alpha$-o-galactopyranosyl-(1 $\rightarrow 4)-2,3,6$-tri-O-benzyl- $\beta$-o- galactopyranosyl-(1 $\rightarrow 4)$-2,3,6-tri-O-benzyl- $\beta$-o-glucopyranoside(17)}

Compound $16(0.51 \mathrm{~g}, 0.34 \mathrm{mmol})$ was dissolved in a mixture of $\mathrm{CH}_{2} \mathrm{Cl}_{2} / \mathrm{H}_{2} \mathrm{O}(4.9 \mathrm{~mL} / 0.5 \mathrm{~mL})$ and the solution was cooled to $0{ }^{\circ} \mathrm{C}$. DDQ $(0.84 \mathrm{~g}, 0.37 \mathrm{mmol})$ was added and the mixture was stirred at room temperature for 4 hours. The mixture was filtered, diluted with $\mathrm{CH}_{2} \mathrm{Cl}_{2}(100$ $\mathrm{mL}$ ) and the organic phase was washed with $\mathrm{H}_{2} \mathrm{O}$ until the solution became colorless. Silica gel column chromatography (4:1 Hexanes-EtOAc) afforded compound $\mathbf{1 7}$ as white solid (0.37 $\mathrm{g}, 80 \%) \cdot[\alpha]_{\mathrm{D}}+34.6\left(c=1, \mathrm{CH}_{2} \mathrm{Cl}_{2}\right) ;{ }^{1} \mathrm{H}-\mathrm{NMR}\left(600 \mathrm{MHz}, \mathrm{CDCl}_{3}\right): \boldsymbol{\delta} 1.87(\mathrm{~m}, 2 \mathrm{H}$, $\mathrm{CH}_{2} \mathrm{CH}_{2} \mathrm{CH}_{2}$ ), 3.17 (dd, $1 \mathrm{H}, J=4.8,8.4 \mathrm{~Hz}, \mathrm{H}-6 \mathrm{a}$ ), 3.27-3.39 (m, 6H, H-2', H-3, H-5, H-5', $\mathrm{OCH}_{2}$ ), 3.44-3.62 (m, 6H, H-2, H-3', H-5", H-6b, H-6a', $\mathrm{CH}_{2} \mathrm{~N}_{3}$ ), 3.69-3.82 (m, 3H, H-2", H6a", H-6b'), 3.91-4.14 (m, 9H, H-4, H-4', H-4", H-3", $\mathrm{CH}_{2} \mathrm{~N}_{3}, \mathrm{CH}_{2} \mathrm{Ph}$ ), 4.34-4.39 (m, 3H, $\left.J_{1^{\prime}, 2^{\prime}}=7.8 \mathrm{~Hz}, \mathrm{H}-1^{\prime}, \mathrm{H}-6 \mathrm{a}^{\prime}, \mathrm{CH}_{2} \mathrm{Ph}\right), 4.45\left(\mathrm{~d}, 1 \mathrm{H}, J_{1,2}=7.8 \mathrm{~Hz}, \mathrm{H}-1\right), 4.47-4.54(\mathrm{~m}, 4 \mathrm{H}$, $\left.\mathrm{CH}_{2} \mathrm{Ph}\right), 4.67-4.84\left(\mathrm{~m}, 8 \mathrm{H}, \mathrm{CH}_{2} \mathrm{Ph}\right), 5.07\left(\mathrm{~d}, 1 \mathrm{H}, J=12.0 \mathrm{~Hz}, \mathrm{CH}_{2} \mathrm{Ph}\right), 5.10\left(\mathrm{~d}, 1 \mathrm{H}, J_{1 ", 2}{ }^{\prime \prime}=\right.$ $3.0 \mathrm{~Hz}, \mathrm{H}-1$ "), $7.13-7.39$ (m, 45H, aromatic); ${ }^{13} \mathrm{CNMR}\left(150 \mathrm{MHz}, \mathrm{CDCl}_{3}\right): \delta$ 29.5, 48.6, 66.8, $67.9,68.0,68.5,69.5,70.3,72.4,73.27,73.33,73.38,73.43,75.1,75.31,75.36,75.47,75.51$, 75.53, 77.2, 77.9, 79.6, 81.8, 81.9, 83.2, 89.5, 99.9, 103.0, 103.8, 127.5, 127.74, 127.76, 127.78, $127.79,127.85,127.86,127.88,127.92,127.95,128.00,128.02,128.10,128.27,128.34$, $128.35,128.42,128.44,128.52,128.55,128.56,128.58,128.60,128.71,128.79,138.27$, 
138.48, 138.57, 138.62, 138.82, 138.83, 138.93, 139.02, 139.6; HRMS: [M+Na] ${ }^{+}$

$\mathrm{C}_{84} \mathrm{H}_{91} \mathrm{~N}_{3} \mathrm{NaO}_{16}$ calcd 1420.6297, obsd 1420.6276.

\section{p-Tolyl 2,3,4-tri-O-benzyl-1-thio- $\beta$---fucopyranoside (18)}

L-Fucose $(10 \mathrm{~g}, 60.9 \mathrm{mmol})$ and DMAP $(0.72 \mathrm{~g}, 6.01 \mathrm{mmol})$ were dissolved in anhydrous pyridine $(100 \mathrm{~mL})$ and acetic anhydride $(40 \mathrm{~mL})$ was added at room temperature in a period of 30 minutes. The mixture was stirred at room temperature under $\mathrm{N}_{2}$ overnight and then quenched with ethanol $(20 \mathrm{~mL})$ at $0{ }^{\circ} \mathrm{C}$. The mixture was concentrated and the resulting residue was diluted with ethyl acetate $(300 \mathrm{~mL})$, washed with water, saturated $\mathrm{NaHCO}_{3}, 10 \%$ aqueous hydrochloric acid and brine. The organic phase was dried over $\mathrm{Na}_{2} \mathrm{SO}_{4}$ and then filtered and concentrated. Silica gel column chromatography (2:1 Hexanes-EtOAc) afforded the 1,2,3,4tetra- $O$-acetyl-L-fucopyranoside as white solid ( $21 \mathrm{~g}$, quantitative, $\alpha / \beta$ mixtures) with the $\alpha$ isomer (S16) as the major product. ${ }^{1} \mathrm{H}-\mathrm{NMR}\left(600 \mathrm{MHz}, \mathrm{CDCl}_{3}\right): \delta 1.01\left(\mathrm{~d}, 3 \mathrm{H}, J_{5,6}=6.6 \mathrm{~Hz}\right.$, $\mathrm{H}-6), 1.86,1.87,2.01,2.04\left(4 \mathrm{~s}, 12 \mathrm{H}, 4 \times \mathrm{COCH}_{3}\right), 4.15(\mathrm{~m}, 1 \mathrm{H}, \mathrm{H}-5), 5.15\left(\mathrm{~d}, 1 \mathrm{H}, J_{1,2}=3.6\right.$ $\mathrm{Hz}, \mathrm{H}-2), 5.16-5.20$ (m, 2H, H-3, H-4), 6.18 (d, $\left.1 \mathrm{H}, J_{1,2}=3.6 \mathrm{~Hz}, \mathrm{H}-1\right) ;{ }^{13} \mathrm{C}-\mathrm{NMR}(150 \mathrm{MHz}$, $\left.\mathrm{CDCl}_{3}\right): \delta$ 16.0, 20.6, 20.7, 20.8, 21.0, 66.6, 67.3, 67.9, 70.6, 89.9, 169.2, 170.0, 170.2, 170.6. ESI-MS $[\mathrm{M}+\mathrm{Na}]^{+} \mathrm{C}_{14} \mathrm{H}_{20} \mathrm{NaO}_{9}$ calcd 355.1 , obsd 355.3. The obtained $\alpha / \beta$ mixture of $1,2,3,4-$ tetra- $O$-acetyl-ı-fucopyranoside $(21 \mathrm{~g}), p$-toluenethiol $(8.32 \mathrm{~g}, 67 \mathrm{mmol})$ were dissolved in $\mathrm{CH}_{2} \mathrm{Cl}_{2}(180 \mathrm{~mL})$ and cooled to $0{ }^{\circ} \mathrm{C}$. Boron trifluoride etherate $(10.5 \mathrm{~mL}, 83 \mathrm{mmol})$ was added dropwise at $0{ }^{\circ} \mathrm{C}$ and the mixture was stirred under $\mathrm{N}_{2}$ at room temperature overnight. The mixture was diluted with $\mathrm{CH}_{2} \mathrm{Cl}_{2}$ and washed with saturated aqueous $\mathrm{NaHCO}_{3}$ until the $\mathrm{pH}$ around 7 and then dried over $\mathrm{Na}_{2} \mathrm{SO}_{4}$, filtered and concentrated. The obtained crude product was recrystallized by EtOAc/hexanes to give $p$-tolyl 2,3,4-tri- $O$-acetyl-1-thio- $\beta$-L-

fucopyranoside ${ }^{63}$ (S17) as white solid $(11.6 \mathrm{~g}, 48 \%) .{ }^{1} \mathrm{H}-\mathrm{NMR}\left(600 \mathrm{MHz}, \mathrm{CDCl}_{3}\right): \boldsymbol{\delta} 1.15(\mathrm{~d}$, $\left.3 \mathrm{H}, J_{5,6}=6.6 \mathrm{~Hz}, \mathrm{H}-6\right), 1.90,2.01,2.07\left(3 \mathrm{~s}, 9 \mathrm{H}, 3 \times \mathrm{COCH}_{3}\right), 2.26\left(\mathrm{~s}, 3 \mathrm{H}, \mathrm{SPhCH}_{3}\right), 3.74(\mathrm{~m}$, $1 \mathrm{H}, \mathrm{H}-5), 4.58\left(\mathrm{~d}, 1 \mathrm{H}, J_{1,2}=10.2 \mathrm{~Hz}, \mathrm{H}-1\right), 4.98\left(\mathrm{dd}, 1 \mathrm{H}, J_{2,3}=9.6 \mathrm{~Hz}, J_{3,4}=3.0 \mathrm{~Hz}, \mathrm{H}-3\right)$, $5.13(\mathrm{t}, 1 \mathrm{H}, J=9.6 \mathrm{~Hz}, \mathrm{H}-2), 5.18\left(\mathrm{~d}, 1 \mathrm{H}, J_{3,4}=3.0 \mathrm{~Hz}, \mathrm{H}-4\right), 7.00-7.09(\mathrm{~m}, 2 \mathrm{H}), 7.30-7.39$ $(\mathrm{m}, 2 \mathrm{H}) ;{ }^{13} \mathrm{C}-\mathrm{NMR}\left(150 \mathrm{MHz}, \mathrm{CDCl}_{3}\right): \boldsymbol{\delta} 16.7,20.8,20.9,21.1,21.3,67.5,70.5,72.6,73.2$, 86.9, 129.3, 129.8, 133.0, 138.3, 169.6, 170.3, 170.8. ESI-MS $[\mathrm{M}+\mathrm{Na}]^{+} \mathrm{C}_{19} \mathrm{H}_{24} \mathrm{NaO}_{7} \mathrm{~S}$ calcd 419.1, obsd 419.2. $p$-tolyl 2,3,4-tri- $O$-acetyl-1-thio- $\beta$-- -fucopyranoside $\mathrm{S} 17$ (9.2 g, $23.2 \mathrm{mmol}$ ) was dissolved in a mixture of $\mathrm{CH}_{2} \mathrm{Cl}_{2} / \mathrm{MeOH}(70 \mathrm{~mL} / 50 \mathrm{~mL})$ and $1 \mathrm{M} \mathrm{NaOMe}(12 \mathrm{~mL}, 11.6$ $\mathrm{mmol}$ ) was added at room temperature. The mixture was stirred for $2 \mathrm{~h}$ at room temperature under $\mathrm{N}_{2}$, neutralized with Amberlite IR-120, concentrated and vacuum dried. The obtained residue $(6 \mathrm{~g})$ was dissolved in DMF $(100 \mathrm{~mL})$ and cooled to $0{ }^{\circ} \mathrm{C}$. $\mathrm{NaH}(3.6 \mathrm{~g}, 60 \% \mathrm{NaH}$ in mineral oil, $88 \mathrm{mmol})$ was added in portions, followed by addition of $\mathrm{BnBr}(10.5 \mathrm{~mL}, 88 \mathrm{mmol})$ 30 minutes later. The mixture was stirred at room temperature under $\mathrm{N}_{2}$ for 2 hours and then diluted with EtOAc $(300 \mathrm{~mL})$. The mixture was washed with saturated aqueous solution of $\mathrm{NaHCO}_{3}$, water and then dried over $\mathrm{Na}_{2} \mathrm{SO}_{4}$, filtered and concentrated. The obtained crude product was recrystallized by EtOAc/Hexanes to give compound $\mathbf{1 8}^{37}$ as white solid (9.4 g, $75 \%$ for two steps); $[\alpha]_{\mathrm{D}}-9.4\left(c=1, \mathrm{CH}_{2} \mathrm{Cl}_{2}\right) ;{ }^{1} \mathrm{H}-\mathrm{NMR}\left(600 \mathrm{MHz}, \mathrm{CDCl}_{3}\right): \boldsymbol{\delta} 1.25(\mathrm{~d}, 3 \mathrm{H}$, $\left.J_{5,6}=6.6 \mathrm{~Hz}, \mathrm{H}-6\right), 2.29\left(\mathrm{~s}, 3 \mathrm{H}, \mathrm{SPhCH}_{3}\right), 3.50(\mathrm{~m}, 1 \mathrm{H}, \mathrm{H}-5), 3.58\left(\mathrm{dd}, 1 \mathrm{H}, J_{2,3}=9.6 \mathrm{~Hz}\right.$, $\left.J_{3,4}=2.4 \mathrm{~Hz}, \mathrm{H}-3\right), 3.62\left(\mathrm{~d}, 1 \mathrm{H}, J_{3,4}=2.4 \mathrm{~Hz}, \mathrm{H}-4\right), 3.89(\mathrm{t}, 1 \mathrm{H}, J=9.6 \mathrm{~Hz}, \mathrm{H}-2), 4.55(\mathrm{~d}, 1 \mathrm{H}$, $\left.J_{1,2}=10.2 \mathrm{~Hz}, \mathrm{H}-1\right), 4.66\left(\mathrm{~d}, 1 \mathrm{H}, J=12.0 \mathrm{~Hz}, \mathrm{CH}_{2} \mathrm{Ph}\right), 4.72-4.81(\mathrm{~m}, 4 \mathrm{H}, J=12.0 \mathrm{~Hz}$, $\left.\mathrm{CH}_{2} \mathrm{Ph}\right), 5.00\left(\mathrm{~d}, 1 \mathrm{H}, J=12.0 \mathrm{~Hz}, \mathrm{CH}_{2} \mathrm{Ph}\right), 6.94-7.08(\mathrm{~m}, 2 \mathrm{H}), 7.27-7.40$ (m, 15H, aromatic), 7.44-7.56 (m, 2H); ${ }^{13} \mathrm{C}-\mathrm{NMR}\left(150 \mathrm{MHz}, \mathrm{CDCl}_{3}\right): \delta$ 17.6, 21.4, 73.1, 74.8, 75.8, 76.8, 77.4, $84.8,88.1,127.7,127.8,127.95,127.97,128.2,128.4,128.59,128.62,128.70,129.78,130.7$, 132.4, 137.4, 138.6, 138.7, 139.0. ESI-MS $[\mathrm{M}+\mathrm{Na}]^{+} \mathrm{C}_{34} \mathrm{H}_{36} \mathrm{NaO}_{4} \mathrm{~S}$ calcd 563.2, obsd 563.5.

\section{3-Azidopropyl 2,3,4-tri-O-benzyl- $\alpha$-L-fucopyranosyl-(1 $\rightarrow 2)-3,4,6$-tri- $O$-benzyl- $\beta$-o- galactopyranosyl-(1 $\rightarrow 3)-4,6$-benzylidene-2-(2,2,2-tri-chloroethoxycarbonylamino)-2-deoxy- $\beta$-o-galactopyranosyl-(1 $\rightarrow 3)-2,4,6$-tri- $O$-benzyl- $\alpha$-o-galactopyranosyl-(1 $\rightarrow 4)-2,3,6$-tri- $O$ - benzyl- $\beta$-o-galactopyranosyl-(1 $\rightarrow 4)-2,3,6$-tri-O-benzyl- $\beta$-o-glucopyranoside $(20 \alpha)$ and 3-}




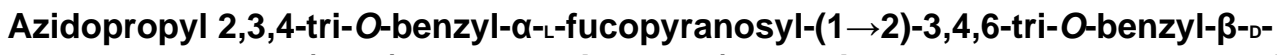 galactopyranosyl-(1 $\rightarrow 3)-4,6$-benzylidene-2-(2,2,2-tri-chloroethoxycarbonylamino)-2-deoxy- $\beta$-o-galactopyranosyl-(1 $\rightarrow 3$ )-2,4,6-tri- $O$-benzyl- $\beta$-o-galactopyranosyl-(1 $\rightarrow 4)-2,3,6$-tri- $O$ - benzyl- $\beta$-o-galactopyranosyl-( $1 \rightarrow 4)-2,3,6$-tri- $O$-benzyl- $\beta$-o-glucopyranoside $(20 \beta)$}

After the donor 18 (50 mg, $92.47 \mu \mathrm{mol})$ and freshly activated molecular sieve MS-4 A (500 $\mathrm{mg}$ ) were stirred for 30 minutes at room temperature in $\mathrm{Et}_{2} \mathrm{O}(4 \mathrm{~mL})$, the solution was cooled to $-78^{\circ} \mathrm{C}$, followed by addition of $\mathrm{AgOTf}(72 \mathrm{mg}, 277.4 \mu \mathrm{mol})$ in $\mathrm{Et}_{2} \mathrm{O}(1.5 \mathrm{~mL})$. The mixture was stirred for 5 minutes at $-78{ }^{\circ} \mathrm{C}$ and then $p$ - TolSCl $(14.7 \mu \mathrm{L}, 92.47 \mu \mathrm{mol})$ was added into the solution. (See the general procedure for single step pre-activation based glycosylation for precautions) The mixture was vigorously stirred for 5 minutes, followed by addition of a solution of acceptor $13(77.1 \mathrm{mg}, 78.60 \mu \mathrm{mol})$ and TTBP $(23 \mathrm{mg}, 92.47 \mu \mathrm{mol})$ in $\mathrm{CH}_{2} \mathrm{Cl}_{2}$ (1 $\mathrm{mL}$ ). The reaction mixture was stirred for 2 hour from -78 to $-20{ }^{\circ} \mathrm{C}$ and then the mixture was cooled down to $-78{ }^{\circ} \mathrm{C}$, followed by addition of $\operatorname{AgOTf}(24 \mathrm{mg}, 92.47 \mu \mathrm{mol})$ in $\mathrm{Et}_{2} \mathrm{O}(1 \mathrm{~mL})$. The mixture was stirred for 10 minutes at $-78^{\circ} \mathrm{C}$ and then $p$ - TolSCl $(12.5 \mu \mathrm{L}, 78.60 \mu \mathrm{mol})$ was added into the solution. After stirred for 5 minutes, a solution of acceptor 19 (30.9 mg, $55.48 \mu \mathrm{mol})$ and TTBP $(23 \mathrm{mg}, 92.47 \mu \mathrm{mol})$ in $\mathrm{CH}_{2} \mathrm{Cl}_{2}(1 \mathrm{~mL})$ was added slowly along the flask wall into the mixture and the reaction mixture was stirred for $2 \mathrm{~h}$ from -78 to $-20{ }^{\circ} \mathrm{C}$. The mixture was cooled down again to $-78^{\circ} \mathrm{C}$, followed by sequential additions of AgOTf $(24 \mathrm{mg}, 92.47 \mu \mathrm{mol})$ in $\mathrm{Et}_{2} \mathrm{O}(1 \mathrm{~mL})$, the last acceptor $15(35.7 \mathrm{mg}, 36.99 \mu \mathrm{mol})$ and TTBP (23 mg, $92.47 \mu \mathrm{mol})$ in $\mathrm{CH}_{2} \mathrm{Cl}_{2}(1 \mathrm{~mL})$. The mixture was stirred for 10 minutes at $-78{ }^{\circ} \mathrm{C}$ and then $p$ - TolSCl $(12.5 \mu \mathrm{L}, 78.60 \mu \mathrm{mol})$ was added into the solution. The reaction mixture was stirred for 2 hours from -78 to $10{ }^{\circ} \mathrm{C}$ and then was quenched with $\mathrm{Et}_{3} \mathrm{~N}(40 \mu \mathrm{L})$, concentrated under vacuum to dryness. The resulting residue was diluted with $\mathrm{CH}_{2} \mathrm{Cl}_{2}(30 \mathrm{~mL})$, followed by filtration. The organic phase was washed with saturated aqueous solution of $\mathrm{NaHCO}_{3}$, $\mathrm{H}_{2} \mathrm{O}$ and then dried over $\mathrm{Na}_{2} \mathrm{SO}_{4}$, filtered and concentrated. Silica gel column chromatography (2:1 Hexanes-EtOAc) afforded $46.6 \mathrm{mg}$ of $\mathbf{2 0 \alpha}(47 \%)$ and $22.4 \mathrm{mg}$ of $\mathbf{2 0 \beta}(23 \%)$ respectively as colorless gel. For $\mathbf{2 0 \alpha}:[\boldsymbol{\alpha}]_{\mathrm{D}}-18.0\left(c=2.4, \mathrm{CH}_{2} \mathrm{Cl}_{2}\right) ;{ }^{1} \mathrm{H}-\mathrm{NMR}\left(600 \mathrm{MHz}, \mathrm{CDCl}_{3}\right): \boldsymbol{\delta} 0.42$ (d, $3 \mathrm{H}, J=6.6 \mathrm{~Hz}, \mathrm{H}-6$ '"' '), $1.84\left(\mathrm{~m}, 2 \mathrm{H}, \mathrm{OCH}_{2} \mathrm{CH}_{2} \mathrm{CH}_{2} \mathrm{~N}_{3}\right), 2.80(\mathrm{~s}, 1 \mathrm{H}), 3.19(\mathrm{~m}, 1 \mathrm{H}), 3.27-$ $3.39(\mathrm{~m}, 7 \mathrm{H}), 3.46-3.68(\mathrm{~m}, 11 \mathrm{H}), 3.76-3.86(\mathrm{~m}, 5 \mathrm{H}), 3.92-4.25(\mathrm{~m}, 17 \mathrm{H}), 4.29-4.59(\mathrm{~m}$, $18 \mathrm{H}), 4.64-4.87(\mathrm{~m}, 13 \mathrm{H}), 4.94\left(\mathrm{~d}, 1 \mathrm{H}, J_{1}{ }^{\prime}, 2^{\prime}=3.0 \mathrm{~Hz}, \mathrm{H}-1\right.$ ”), $5.06(\mathrm{~d}, 1 \mathrm{H}, J=11.4 \mathrm{~Hz}), 5.24$ $(\mathrm{d}, 1 \mathrm{H}, J=11.4 \mathrm{~Hz}), 5.42(\mathrm{~s}, 1 \mathrm{H}), 5.55$ (d, $1 \mathrm{H}, J_{1}{ }^{\prime},{ }^{\prime}, 2^{\prime}, ",=3.6 \mathrm{~Hz}, \mathrm{H}-1$ '”'”), 7.00-7.45 (m, 80H, aromatic); ${ }^{13} \mathrm{C}-\mathrm{NMR}\left(150 \mathrm{MHz}, \mathrm{CDCl}_{3}\right): \delta \mathrm{\delta} 16.1,29.4,29.9,48.5,54.4,66.2,66.6,66.7,67.7$, 68.1, 68.4, 69.0, 69.2, 69.4, 72.0, 72.1, 72.5, 72.7, 72.9, 73.1, 73.22, 73.24, 73.28, 73.57, 73.63, 73.77, 73.9, 74.57, 74.67, 74.76, 74.78, 74.94, 75.13, 75.18, 75.27, 75.4, 76.2, 76.3, 76.8, 77.6, 78.6, 79.2, 79.7, 80.4, 81.5, 81.7, 82.0, 84.1, 95.8, 97.5, 100.8, 101.8, 102.6, 102.7, 102.8, 103.6, 126.6, 127.03, 127.06, 127.24, 127.28, 127.31, 127.41, 127.44, 127.55, 127.61, 127.67, $127.68,127.70,127.76,127.79,127.81,127.93,128.01,128.02,128.11,128.16,128.18$, $128.20,128.34,128.38,128.42,128.45,128.50,128.56,128.65,128.68,128.72,128.76$, $128.77,129.1,129.9,137.9,138.2,138.3,138.44,138.45,138.47,138.67,138.81,138.94$, 138.99, 139.1, 139.4, 139.7, 139.8, 154.0; HRMS: $[\mathrm{M}+\mathrm{Na}]^{+} \mathrm{C}_{154} \mathrm{H}_{163} \mathrm{Cl}_{3} \mathrm{~N}_{4} \mathrm{O}_{31}$ calcd 2692.0265, obsd 2692.0332. gHMQC (without ${ }^{1} \mathrm{H}$ decoupling): ${ }^{1} \mathrm{~J}_{\mathrm{C} 1}{ }^{\prime},{ }^{\prime}, \mathrm{H} 1{ }^{\prime},{ }^{\prime \prime},=171.9$ $\mathrm{Hz},{ }^{1} \mathrm{~J}_{\mathrm{Cl}}{ }^{\prime}, \mathrm{H} 1 "=169.8 \mathrm{~Hz}$, other four ${ }^{1} \mathrm{~J}_{\mathrm{C} 1, \mathrm{H} 1}=160.1 \mathrm{~Hz}, 160.1 \mathrm{~Hz}, 162.6 \mathrm{~Hz}, 162.6 \mathrm{~Hz}$.

For 20ק: $[\boldsymbol{\alpha}]_{\mathrm{D}}-4.2\left(c=1, \mathrm{CH}_{2} \mathrm{Cl}_{2}\right) ;{ }^{1} \mathrm{H}-\mathrm{NMR}\left(600 \mathrm{MHz}, \mathrm{CDCl}_{3}\right): \boldsymbol{\delta} 0.60(\mathrm{~d}, 3 \mathrm{H}, J=6.6 \mathrm{~Hz}$, H-6"”'”), $1.85\left(\mathrm{~m}, 2 \mathrm{H}, \mathrm{OCH}_{2} \mathrm{CH}_{2} \mathrm{CH}_{2} \mathrm{~N}_{3}\right), 3.22-3.63(\mathrm{~m}, 21 \mathrm{H}), 3.66-3.79(\mathrm{~m}, 6 \mathrm{H}), 3.86-3.99$ $(\mathrm{m}, 6 \mathrm{H}), 4.05-4.24(\mathrm{~m}, 7 \mathrm{H}), 4.28-4.51(\mathrm{~m}, 18 \mathrm{H}), 4.55-4.60(\mathrm{~m}, 4 \mathrm{H}), 4.63-4.79(\mathrm{~m}, 8 \mathrm{H}), 4.85-$ $4.94(\mathrm{~m}, 3 \mathrm{H}), 5.09-5.13(\mathrm{~m}, 2 \mathrm{H}), 5.22(\mathrm{~d}, 1 \mathrm{H}, J=12.0 \mathrm{~Hz}), 5.49(\mathrm{~s}, 1 \mathrm{H}), 5.55(\mathrm{~d}, 1 \mathrm{H}$, $J_{1}$ '"', $, 2, ",=3.6 \mathrm{~Hz}, \mathrm{H}-1$ '”,'), 6.92-7.43 (m, 80H, aromatic); ${ }^{13} \mathrm{C}-\mathrm{NMR}\left(150 \mathrm{MHz}, \mathrm{CDCl}_{3}\right): \boldsymbol{\delta}$ $16.4,29.5,29.9,48.5,54.7,66.6,67.1,68.2,68.8,69.0,69.2,69.3,70.0,72.3,72.68,72.74$, 73.0, 73.3, 73.67, 73.69, 73.75, 73.80, 73.96, 74.75, 74.99, 75.12, 75.2, 75.4, 75.8, 76.2, 76.3, 76.6, 78.4, 79.3, 79.9, 80.6, 81.4, 81.8, 82.6, 83.2, 83.7, 96.0, 97.4, 101.6, 101.9, 102.8, 103.1, $103.7,126.8,126.92,126.96,127.1,127.2,127.36,127.40,127.45,127.48,127.51,127.53$,

J Org Chem. Author manuscript; available in PMC 2008 September 11. 
$127.67,127.69,127.73,127.76,127.83,127.87,127.90,127.93,128.01,128.07,128.10$, $128.16,128.27,128.28,128.34,128.35,128.38,128.44,128.50,128.52,128.53,128.60$, $128.64,128.67,128.71,129.2,138.1,138.2,138.3,138.43,138.47,138.51,138.57,138.86$, 138.88, 138.97, 139.06, 139.24, 139.51, 139.8, 140.0, 154.1; HRMS: $[\mathrm{M}+\mathrm{Na}]^{+}$ $\mathrm{C}_{154} \mathrm{H}_{163} \mathrm{Cl}_{3} \mathrm{~N}_{4} \mathrm{NaO}_{31}$ calcd 2692.0265, obsd 2692.0254. ${ }^{1} \mathrm{~J}_{\mathrm{Cl}}$ '”,',H1",", $=170.9 \mathrm{~Hz}$, other five ${ }^{1} \mathrm{~J}_{\mathrm{C} 1, \mathrm{H} 1}=162.0 \mathrm{~Hz}, 162.0 \mathrm{~Hz}, 162.0 \mathrm{~Hz}, 162.0 \mathrm{~Hz} .159 .4 \mathrm{~Hz}$.

Three component one-pot synthesis procedure:

After the donor 18 (50 mg, $92.47 \mu \mathrm{mol})$ and activated molecular sieve MS-4 $\AA$ (500 mg) were stirred for 30 minutes at room temperature in $\mathrm{Et}_{2} \mathrm{O}(6 \mathrm{~mL})$, the solution was cooled to $-78^{\circ}$ $\mathrm{C}$, followed by addition of $\mathrm{AgOTf}(72 \mathrm{mg}, 277.4 \mu \mathrm{mol})$ in $\mathrm{Et}_{2} \mathrm{O}(1.5 \mathrm{~mL})$. The mixture was stirred for 5 minutes at $-78^{\circ} \mathrm{C}$ and then $p$-TolSCl $(14.7 \mu \mathrm{L}, 92.47 \mu \mathrm{mol})$ was added into the solution. (See the general procedure for single step pre-activation based glycosylation for precautions) The mixture was vigorously stirred for 10 minutes, followed by addition of a solution of acceptor $13(81.7 \mathrm{mg}, 83.22 \mu \mathrm{mol})$ and TTBP $(23 \mathrm{mg}, 92.47 \mu \mathrm{mol})$ in $\mathrm{CH}_{2} \mathrm{Cl}_{2}(1$ $\mathrm{mL}$ ). The reaction mixture was stirred for 2 hours from -78 to $-20^{\circ} \mathrm{C}$ and then the mixture was cooled down to $-78{ }^{\circ} \mathrm{C}$, followed by sequential additions of AgOTf ( $24 \mathrm{mg}, 92.47 \mu \mathrm{mol}$ ) in $\mathrm{Et}_{2} \mathrm{O}(1 \mathrm{~mL})$, acceptor $17(90.5 \mathrm{mg}, 64.73 \mu \mathrm{mol})$ and TTBP $(23 \mathrm{mg}, 92.47 \mu \mathrm{mol})$ in $\mathrm{CH}_{2} \mathrm{Cl}_{2}(1 \mathrm{~mL})$. The mixture was stirred for 5 minutes at $-78^{\circ} \mathrm{C}$ and then $p$ - TolSCl $(13.2 \mu \mathrm{L}$, $83.22 \mu \mathrm{mol})$ was added into the solution. The reaction mixture was stirred for 3 hours from -78 to $10^{\circ} \mathrm{C}$ and then was quenched with $\mathrm{Et}_{3} \mathrm{~N}(40 \mu \mathrm{L})$, concentrated under vacuum to dryness. The resulting residue was diluted with $\mathrm{CH}_{2} \mathrm{Cl}_{2}(30 \mathrm{~mL})$, followed by filtration. The organic phase was washed with saturated aqueous $\mathrm{NaHCO}_{3}, \mathrm{H}_{2} \mathrm{O}$ and then dried over $\mathrm{Na}_{2} \mathrm{SO}_{4}$, filtered and concentrated. Silica gel column chromatography (2:1 Hexanes-EtOAc) afforded $\mathbf{2 0 \alpha}$ as colorless gel (128mg, 74\%).

\section{3-Azidopropyl $\alpha$---fucopyranosyl-(1 $\rightarrow 2)-\beta$-o-galactopyranosyl-(1 $\rightarrow 3)$-2-acetamido-2-deoxy-

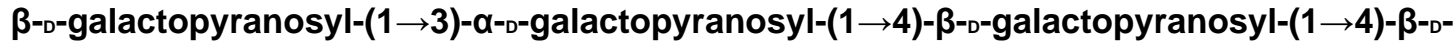 glucopyranoside (2)}

The mixture of compound $\mathbf{2 0 \alpha}(0.075 \mathrm{~g}, 0.028 \mathrm{mmol}), 1 \mathrm{M} \mathrm{NaOH}(0.56 \mathrm{~mL}, 0.56 \mathrm{mmol})$ and THF ( $4 \mathrm{~mL}$ ) was stirred at $50{ }^{\circ} \mathrm{C}$ overnight and then concentrated to dryness. The resulting residue was diluted with $\mathrm{CH}_{2} \mathrm{Cl}_{2}(50 \mathrm{~mL})$ and the organic phase was washed by $\mathrm{H}_{2} \mathrm{O}$ and then dried over $\mathrm{Na}_{2} \mathrm{SO}_{4}$, filtered and concentrated to dryness. The resulting residue was dissolved in pyridine $(2 \mathrm{~mL})$ and catalytic amount of DMAP was added. Acetic anhydride $(0.5 \mathrm{~mL}, 5.3$ mmol) was added dropwise and the mixture was stirred at room temperature under $\mathrm{N}_{2}$ for 4 hours. The reaction was quenched by adding a few drops of $\mathrm{H}_{2} \mathrm{O}$ and then diluted with EtOAc $\left(20 \mathrm{~mL}\right.$ ). The organic phase was washed with saturated aqueous solution of $\mathrm{NaHCO}_{3}, \mathrm{H}_{2} \mathrm{O}$ and then dried over $\mathrm{Na}_{2} \mathrm{SO}_{4}$, filtered and concentrated to dryness. Silica gel column chromatography (2:1 Hexanes-EtOAc) afforded the $N$-acetylation product as white solid. The mixture of the $N$-acetylation product, $1 \mathrm{M}$ of $\mathrm{PMe}_{3}$ in $\mathrm{THF}(56 \mu \mathrm{L}, 0.056 \mathrm{mmol}), 0.1 \mathrm{M} \mathrm{NaOH}$ $(0.5 \mathrm{~mL}, 0.05 \mathrm{mmol})$ and THF $(3 \mathrm{~mL})$ was stirred at $60^{\circ} \mathrm{C}$ under $\mathrm{N}_{2}$ overnight. The mixture was concentrated and the resulting residue was diluted with $\mathrm{CH}_{2} \mathrm{Cl}_{2}(50 \mathrm{~mL})$. The organic phase was washed with $\mathrm{H}_{2} \mathrm{O}$ and then dried over $\mathrm{Na}_{2} \mathrm{SO}_{4}$, filtered and concentrated to dryness. The resulting residue was purified by quickly passing through a short silica gel column (10:1, $\left.\mathrm{CH}_{2} \mathrm{Cl}_{2}-\mathrm{MeOH}\right)$. The mixture of the obtained solid and $\mathrm{Pd}(\mathrm{OH})_{2}$ in $\mathrm{MeOH} / \mathrm{H}_{2} \mathrm{O} / \mathrm{HOAc}$ (3 $\mathrm{mL} / 1 \mathrm{~mL} / 1 \mathrm{~mL}$ ) was stirred under $\mathrm{H}_{2}$ at room temperature overnight and then filtered. The filtrate was concentrated to dryness under vacuum and then was co-evaporated with $\mathrm{H}_{2} \mathrm{O}$ (10 $\mathrm{mL}$ ) three times to remove the $\mathrm{AcOH}$. The aqueous phase was further washed with $\mathrm{CH}_{2} \mathrm{Cl}_{2}(5$ $\mathrm{mL} \times 3)$ and EtOAc $(5 \mathrm{~mL} \times 3)$ and then the aqueous phase was dried under vacuum to afford compound 2 (acetate salt) as white solid $\left(16.2 \mathrm{mg}, 50 \%\right.$ for three steps). $[\alpha]_{\mathrm{D}}+27.8(c=1.4$, $\mathrm{H}_{2} \mathrm{O}$ ); ${ }^{1} \mathrm{H}-\mathrm{NMR}\left(600 \mathrm{MHz}, \mathrm{D}_{2} \mathrm{O}\right): \boldsymbol{\delta} 1.00$ (d, 3H, J = 6.6 Hz, H-6"'”'), 1.74 (s, 3H), 1.79 (m, 
$2 \mathrm{H}), 1.83(\mathrm{~s}, 3 \mathrm{H}), 2.95(\mathrm{t}, 2 \mathrm{H}, J=6.6 \mathrm{~Hz}), 3.12(\mathrm{t}, 1 \mathrm{H}, J=8.4 \mathrm{~Hz}), 3.36-3.89(\mathrm{~m}, 32 \mathrm{H}), 4.02-$ $4.03(\mathrm{~m}, 2 \mathrm{H}), 4.18(\mathrm{t}, 1 \mathrm{H}, J=6.0 \mathrm{~Hz}), 4.29(\mathrm{~d}, 2 \mathrm{H}, J=7.8 \mathrm{~Hz}), 4.32(\mathrm{~d}, 1 \mathrm{H}, J=7.8 \mathrm{~Hz}), 4.40$ $(\mathrm{d}, 1 \mathrm{H}, J=7.8 \mathrm{~Hz}), 4.69(\mathrm{~d}, 1 \mathrm{H}, J=3.6 \mathrm{~Hz}), 5.02(\mathrm{~d}, 1 \mathrm{H}, J=3.6 \mathrm{~Hz}) ;{ }^{13} \mathrm{C}-\mathrm{NMR}(150 \mathrm{MHz}$, $\left.\mathrm{D}_{2} \mathrm{O}\right): \delta$ 15.4, 22.3, 23.4, 26.8, 37.7, 51.8, 60.1, 60.5, 61.1, 66.9, 67.9, 68.1, 68.6, 69.2, 69.3, 69.6, 70.2, 71.0, 72.0, 72.2, 73.0, 73.7, 74.5, 74.7, 75.0, 75.2, 75.6, 76.2, 76.4, 77.3, 78.3, 78.8, 99.4, 100.6, $102.2(\times 2), 103.5,104.1,174.4$; HRMS: $[\mathrm{M}+\mathrm{Na}]^{+} \mathrm{C}_{41} \mathrm{H}_{72} \mathrm{~N}_{2} \mathrm{NaO}_{30}$ calcd

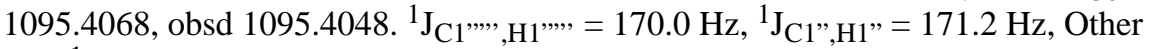
four ${ }^{1} \mathrm{~J}_{\mathrm{C} 1, \mathrm{H} 1}=162.6 \mathrm{~Hz}, 163.9 \mathrm{~Hz}, 162.4 \mathrm{~Hz}, 162.4 \mathrm{~Hz}$.

\section{Supplementary Material}

Refer to Web version on PubMed Central for supplementary material.

\section{Acknowledgment}

We are grateful for financial supports from the University of Toledo, the National Institutes of Health (R01-GM-72667) and the Pardee foundation. We dedicate this work to Prof. Nina Berova, Columbia University, for her guidance and continual encouragements over the years.

\section{References}

1. Zhang S, Zhang HS, Reuter VE, Slovin SF, Scher HI, Livingston PO. Clin. Cancer Res 1998;4:295302. [PubMed: 9516914]

2. Hakomori S, Zhang Y. Chem. Biol 1997;4:97-104. [PubMed: 9190292]

3. Zhang S, Cordon-Cardo C, Zhang HS, Reuter VE, Adluri S, Hamilton WB, Lloyd KO, Livingston PO. Int. J. Cancer 1997;73:42-49. [PubMed: 9334808]

4. Kannagi R, Levery SB, Ishijamik F, Hakomori S, Schevinsky LH, Knowles BB, Solter D. J. Biol. Chem 1983;258:8934-8942. [PubMed: 6863318]

5. Bremer EG, Levery SB, Sonnino S, Ghidoni R, Canevari S, Kannagi R, Hakomori S. J. Biol. Chem 1984;259:14773-14777. [PubMed: 6501317]

6. Gilewske T, Ragupathi G, Bhuta S, Williams LJ, Musselli C, Zhang X-F, Bencsath KP, Panageas KS, Chin J, Hudis CA, Norton L, Houghton AN, Livingston PO, Danishefsky SJ. Proc. Natl. Acad. Sci. U. S. A 2001;98:3270-3275. [PubMed: 11248068]

7. Wang ZG, Williams LJ, Zhang XF, Zatorski A, Kudrya-shov V, Ragupathi G, Spassova M, Bornmann W, Slovin SF, Scher HI, Livingston PO, Lloyd KO, Danishefsky SJ. Proc. Natl. Acad. Sci. U. S. A 2000;97:2719. [PubMed: 10716997]

8. Danishefsky SJ, Allen JR. Angew. Chem. Int. Ed 2000;39:836-863.

9. Slovin SF, Ragupathi G, Adluri S, Ungers G, Terry K, Kim S, Spassova M, Bornmann WG, Fazzari M, Dantis L, Olkiewicz K, Lloyd KO, Livingston PO, Danishefsky SJ, Scher HI. Proc. Natl. Acad. Sci. U. S. A 1999;96:5710. [PubMed: 10318949]

10. Park TY, Kim IJ, Hu S, Bilodeau MT, Randolph JT, Kwon O, Danishefsky SJ. J. Am. Chem. Soc 1996;118:11488-11500.

11. Bilodeau MT, Park TK, Hu S, Randolph JT, Danishefsky SJ, Livingston PO, Zhang S. J. Am. Chem. Soc 1995;117:7840-7841.

12. Allen JR, Allen JG, Zhang X-F, Williams LJ, Zatorski A, Ragupathi G, Livingston PO, Danishefsky SJ. Chem. Eur. J 2000;6:1366-1375.

13. Lassaletta JM, Schmidt RR. Liebigs Ann.Chem 1996:1417-1423.

14. Zhu T, Boons GJ. Angew. Chem. Int. Ed 1999;38:3495-3497.

15. Huang C-Y, Thayer DA, Chang AY, Best M, Hoffmann L, Head S, Wong C-H. Proc. Natl. Acad. Sci. U. S. A 2006;100:15-20. [PubMed: 16373501]

16. Wacowich-Sgarbi, S.; Rabuka, D.; Sgarbi, PWM.; Ichikawa, Y. ACS Symposium Series 873; Synthesis of Carbohydrates through Biotechnology. Wang, PG.; Ichikawa, Y., editors. Washington, DC: American Chemical Society; 2004. p. 23-38.

17. Burkhart F, Zhang Z, Wacowich-Sgarbi S, Wong C-H. Angew. Chem. Int. Ed 2001;40:1274-1277. 
18. Bosse F, Marcauelle LA, Seeberger PH. J. Org. Chem 2002;67:6659-6670. [PubMed: 12227795]

19. Werz DD, Castagner B, Seeberger PH. J. Am. Chem. Soc 2007;129:2770-2771. [PubMed: 17302423]

20. For an example, see: Adinolfi M, Iadonisi A, Ravidá A, Schiattarella M. J. Org. Chem 2005;70:53165319. [PubMed: 15960539]

21. Tanaka H, Matoba N, Takanishi T. Chem. Lett 2005;34:400-401.

22. Huang L, Huang X. Chem. Eur. J 2007;13:529-540.

23. Huang L, Wang Z, Li X, Ye X-S, Huang X. Carbohydr. Res 2006;341:1669-1679. [PubMed: 16442505]

24. Huang X, Huang L, Wang H, Ye X-S. Angew. Chem. Int. Ed 2004;42:5221-5224.

25. Yu B, Yang Z, Cao H. Curr. Org. Chem 2005;9:179-194.and references cited therein

26. Pornsuriyasak P, Demchenko AV. Tetrahedron: Asymmetry 2005;6:433-439.and references cited therein

27. Kanie, O. Carbohydrates in Chemistry and Biology. Ernst, B.; Hart, GW.; Sinay, P., editors. Vol. 1. Weinheim: Wiley-VCH; 2000. p. 407-426.

28. Fraser-Reid B, Wu Z, Udodong UE, Ottosson H. J. Org. Chem 1990;55:6068-6070.

29. Koeller KM, Wong C-H. Chem. Rev 2000;100:4465-4493. [PubMed: 11749355] and references cited therein

30. Tanaka H, Adachi M, Takahashi T. Chem. Eur. J 2005;11:849-862.and references cited therein

31. Tanaka H, Adachi M, Tsukamoto H, Ikeda T, Yamada H, Takahashi T. Org. Lett 2002;4:4213-4216. [PubMed: 12443061]

32. Fraser-Reid B, López JC, Gómez AM, Uriel C. Eur. J. Org. Chem 2004;2004:1387-1395.

33. Fraser-Reid B, López JC, Radhakrishnan KV, Mach M, Schlueter U, Gomez AM, Uriel C. J. Am. Chem. Soc 2002;124:3198-3199. [PubMed: 11916390]

34. Jennings, HJ.; Snood, RK. Neoglycoconjugates, Preparation and Application. Lee, YC.; Lee, RT., editors. San Diego: Academic Press; 1994. p. 325

35. Crich D, Sun S. Tetrahedron 1998;54:8321-8348.

36. Martichonok V, Whitesides GM. J. Org. Chem 1996;61:1702-1706. [PubMed: 11667039]

37. Zhang Z, Ollman IR, Ye X-S, Wischnat R, Baasov T, Wong C-H. J. Am. Chem. Soc 1999;121:734753.

38. Bock K, Pedersen C. J. Chem. Soc. Perkin Trans 1974;2:293-297.

39. Demchenko AV. Synlett 2003:1225-1240.and references cited therein

40. Tokimoto H, Fujimoto Y, Fukase K, Kusumoto S. Tetrahedron Asym 2005;16:441-447.

41. Chiba H, Funasaka S, Mukaiyama T. Bull. Chem. Soc. Jpn 2003;76:1629-1644.

42. Adinolfi M, Barone G, Iadonisi A, Schiattarella M. Tetrahedron Lett 2002;43:5573-5577.

43. Jona H, Mandai H, Chavasiri W, Takeuchi K, Mukaiyama T. Bull. Chem. Soc. Jpn 2002;75:291309.

44. Demchenko AV, Stauch T, Boons G-J. Synlett 1997:818-819.

45. Schmidt RR, Behrendt M, Toepfer A. Synlett 1990:694-696.

46. Kamath VP, Yeske RE, Gregson JM, Ratcliffe RM, Fang YR, Palcic MM. Carbohydr. Res 2004;339:1141-1146. [PubMed: 15063203] and references cited therein

47. Chen L, Zhao X-E, Lai D, Song Z, Kong F. Carbohydr. Res 2006;341:1174-1180. [PubMed: 16630600] and references cited therein

48. Hsieh S-Y, Jan M-D, Patkar LN, Chen C-T, Lin C-C. Carbohydr. Res 2005;340:49-57. [PubMed: 15620666]

49. Wang C, Li Q, Wang H, Zhang L, Ye X-S. Tetrahedron 2006;62:11657-11662.

50. Dohi H, Nishida Y, Takeda T, Kobayashi K. Carbohydr. Res 2002;337:983-989. [PubMed: 12039538]

51. Bhattacharyya S, Magnusson BG, Wellmar U, Nilsson UJ. J. Chem. Soc. Perkin Trans 2001;1:886890.

52. Sarkar AK, Matta KL. Carbohydr. Res 1992;233:245-250. [PubMed: 1344884]

53. Pornsuriyasak P, Demchenko AV. Carbohydr. Res 2006;341:1458-1466. [PubMed: 16643871]

J Org Chem. Author manuscript; available in PMC 2008 September 11. 
54. Crich D, Smith M, Yao Q, Picione J. Synthesis 2001:323-326.

55. To determine the configuration of newly formed glycosyl bonds, we once isolated the intermediate oligosaccharides during one-pot synthesis and confirmed the linkages via NMR analysis.

56. Plante OJ, Palmacci ER, Seeberger PH. Science 2001;291:1523-1527. [PubMed: 11222853]

57. Li J, Wang J, Czyryca PG, Chang H, Orsak TW, Evanson R, Chang C-WT. Org. Lett 2004;6:13811384. [PubMed: 15101747]

58. Cheshev PE, Khatuntseva EA, Gerbst AG, Tsvetkov YE, Shashkov AS, Nifantiev NE. Russ. J. Bioorg. Chem 2003;29:372-381.

59. Mong TK-K, Lee H-K, Duron SG, Wong C-H. Proc. Natl. Acad. Sci. U. S. A 2003;100:797-802. [PubMed: 12552090]

60. Nyffeler PT, Liang C-H, Koeller KM, Wong C-H. J. Am. Chem. Soc 2002;124:10773-10778. [PubMed: 12207533]

61. Luening B, Norberg T, Tejbrant J. Glycoconj. J 1989;6:5-19. [PubMed: 2535477]

62. Lee J-C, Wu C-Y, Apon JV, Siuzdak G, Wong CH. Angew. Chem. Int. Ed 2006;45:2753-2757.

63. Tai C-A, Kulkarni SS, Hung S-C. J. Org. Chem 2003;68:8719-8722. [PubMed: 14575510]

64. Yang Z-Q, Puffer EB, Pontrello JK, Kiessling LL. Carbohydr. Res 2002;337:1605-1613. [PubMed: 12423961] 
1) $\mathrm{NaOMe}, \mathrm{MeOH} / \mathrm{CH}_{2} \mathrm{Cl}_{2}$

a) 2) 1,3-propanedithiol, $\mathrm{CH}_{2} \mathrm{Cl}_{2} / \mathrm{MeOH}, \mathrm{Et}_{3} \mathrm{~N}$ $12 \beta$

3) $\mathrm{TrocCl}, \mathrm{NaHCO}_{3}, \mathrm{THF}$

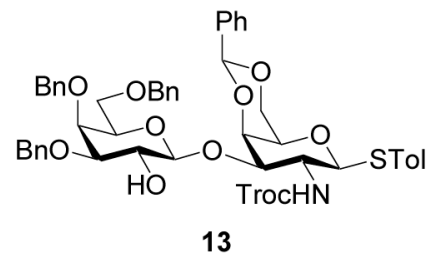

b)

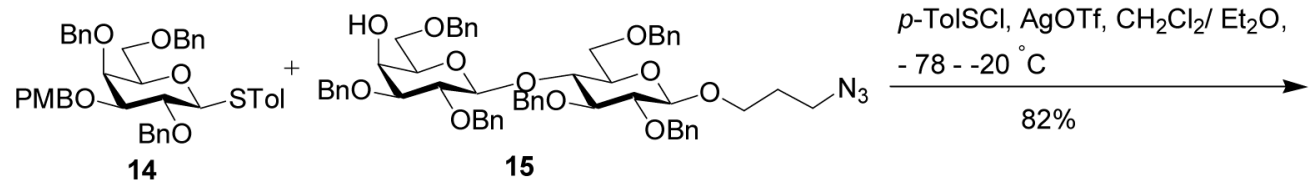

BnO

16: $\mathrm{R}=\mathrm{PMB}$ 2,3-dichloro-5,6-dicyano-1,4-benzoquinone

$\mathrm{BnO}$ O_OBn 17: $\mathrm{R}=\mathrm{H} \quad \mathrm{CH}_{2} \mathrm{Cl}_{2} / \mathrm{H}_{2} \mathrm{O}$

$\mathrm{BnO} \sim 2$

$\Gamma^{\mathrm{OBn}}$

$80 \%$

Scheme 1. 


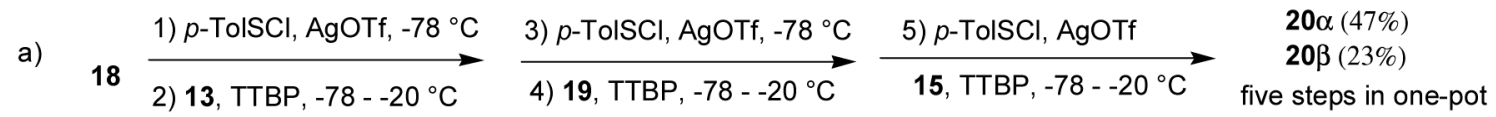

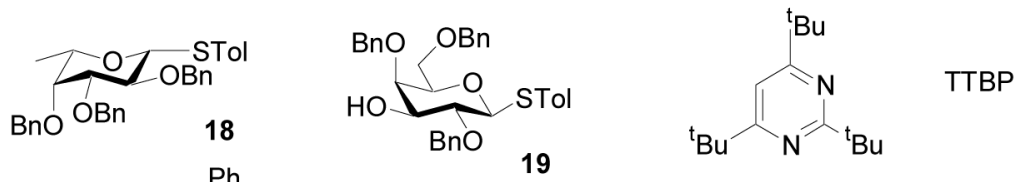
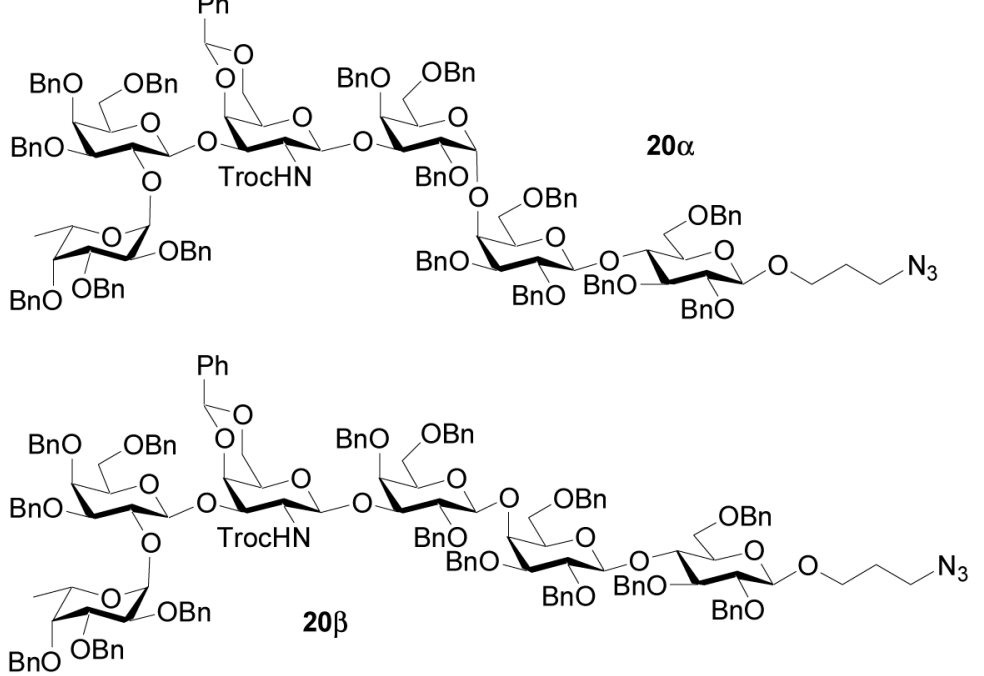

b)

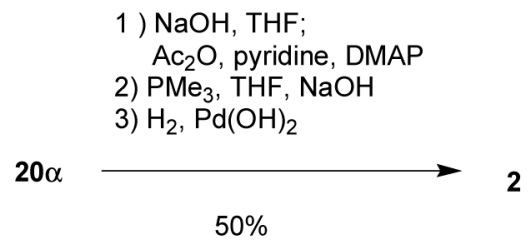

Scheme 2. 


$$
\begin{aligned}
& \text { 1) } p \text {-TolSCl, AgOTf, }-78{ }^{\circ} \mathrm{C} \quad \text { 3) } p \text {-TolSCl, AgOTf } 20 \alpha(74 \%) \\
& 18 \underset{17, \text { TTBP, }-78-10{ }^{\circ} \mathrm{C}}{\longrightarrow} \begin{array}{c}
20 \alpha, \text { TTBP, }-78--20{ }^{\circ} \mathrm{C} \\
\text { three steps in one-pot }
\end{array}
\end{aligned}
$$

Scheme 3. 
Table 1

Evaluation of buiding blocks for $\mathbf{B C}$ disaccharide synthesis.

\begin{tabular}{|c|c|c|c|}
\hline \multirow{2}{*}{ Donor (1 eq) + AgOTf } & \multicolumn{3}{|l|}{ 1) $p$-TolsCl, $-78^{\circ} \mathrm{C}$} \\
\hline & \multicolumn{3}{|l|}{ 2) acceptor (0.9 eq) } \\
\hline Entry \# & Donor & Acceptor & Product (yield) \\
\hline 1 & 3 & 4 & - \\
\hline 2 & 7 & 4 & $9(10 \%)^{a}$ \\
\hline 3 & 8 & 4 & $10(30 \%)^{a}$ \\
\hline 4 & 8 & 6 & $11(30 \%)^{a}$ \\
\hline 5 & 7 & 5 & $12 \beta(50-70 \%)^{a}$ \\
\hline 6 & 8 & 6 & $12 \alpha(10-20 \%)^{a}$ \\
\hline
\end{tabular}

${ }^{[a]} \mathrm{AgOTf}$ was added as a solution in diethyl ether to donor dissolved in dichloromethane. Ratio of diethyl ether to dichloromethane is 1:2

${ }^{[b]} \mathrm{AgOTf}$ was added as a solution in acetonitrile to donor dissolved in dichloromethane. Ratio of acetonitrile to dichloromethane is 1:20. 